\title{
Off-Fault Secondary Failure Induced by a Dynamic Slip Pulse
}

\author{
by James R. Rice, Charles G. Sammis, and Robert Parsons*
}

\begin{abstract}
We develop a 2D slip-weakening description of a self-healing slip pulse that propagates dynamically in a steady-state configuration. The model is used to estimate patterns of off-fault secondary failure induced by the rupture, and also to infer fracture energies $G$ for large earthquakes. This extends an analysis for a semiinfinite rupture (Poliakov et al., 2002) to the case of a finite slipping zone length $L$ of the pulse. The dynamic stress drop, when divided by the drop from peak to residual strength, determines the ratio of $L$ to the slip-weakening zone length $R$. Predicted off-fault damage is controlled by that scaled stress drop, static and dynamic friction coefficients, rupture velocity, principal prestress orientation, and poroelastic Skempton coefficient. All damage zone lengths can be scaled by $R_{o}^{*}$, which is proportional $G /$ (strength drop) $^{2}$ and is the value of $R$ in the low-rupture-velocity, low-stress-drop, limit. In contrast to the Poliakov et al. (2002) case $R / L=0$, the region that supports Coulomb failure reaches a maximum size on the order of $R_{o}^{*}$ when mode II rupture speed approaches the Rayleigh speed. Analysis of slip pulses documented by Heaton (1990) leads to estimates of $G$, each with a factor-of-two model uncertainty, from 0.1 to $9 \mathrm{MJ} / \mathrm{m}^{2}$ (including the factor), averaging $2-4 \mathrm{MJ} / \mathrm{m}^{2}$; $G$ tends to increase with the amount of slip in the event. In most cases, secondary faulting should extend, at high rupture speeds, to distances from the principal fault surface on the order of 1 to $2 R_{o}^{*} \approx 1-80 \mathrm{~m}$ for a $100-\mathrm{MPa}$ strength drop; that distance should vary with depth, being larger near the surface. We also discuss gouge and damage processes.
\end{abstract}

\section{Introduction}

A well-known characteristic of elastodynamic fracture propagation is the strong growth of off-plane stresses near the crack tip, relative to those on the primary fracture plane, at high rupture velocity. This is a feature not only of tensile cracks (Yoffe, 1951) but also of shear ruptures (Erdogan, 1968; Andrews, 1976a; Rice, 1980; Kostrov, orally reported results [1978] cited in Rice, 1980; Kame and Yamashita, 1999). When the shear rupture velocity $v_{r}$ approaches its "limiting speed" $c_{\text {lim }}$ (the shear speed $c_{s}$ for mode III and the Rayleigh speed $c_{R} \approx 0.92 c_{s}$ for mode II), these stresses grow to the point where branching should be expected (Poliakov et al., 2002; Kame et al., 2003). Such branching had been studied earlier on the basis of static crack models (Segall and Pollard, 1980; Pollard and Segall, 1987).

Poliakov et al. (2002) (subsequently referred to as "PDR") calculated the off-fault stresses for dynamic mode II and mode III ruptures in the context of earthquake mechanics and discussed several examples of the general observation that a major earthquake rarely propagates along a single fault plane. More often the propagation path bends to follow

*Present address: Axiam, Inc., 1 Blackburn Center, Gloucester, Massachusetts 01930. an intersecting fault plane or jumps (Harris and Day, 1993) to an offset fault segment. PDR explored the conditions for which the elastodynamic stress field for a singular crack leads to Coulomb failure on intersecting faults, thereby supporting out-of-plane propagation. They also analyzed offfault stressing near a nonsingular crack governed by a slipweakening model, building on the work of Rice (1980) and Rubin and Parker (1994). Their results may be summarized as follows. As expected, stresses that can initiate failure on off-plane secondary faults grow dramatically as $v_{r} \rightarrow c_{\text {lim }}$. However, the continued propagation of off-plane secondary ruptures to distances significantly beyond the crack tip depends on the initial stress field, specifically on its principal stress directions and their ratio. Such predictions for choice of the rupture path along branched-fault systems are quantified in studies by Kame et al. (2003), using a dynamic boundary integral equation formulation for simulation of slip-weakening rupture. They compare favorably with field examples. In mode II, it is very common for off-plane rupture to be encouraged near the crack tip but discouraged at larger distances, which leads to intermittent propagation and/ or arrest on the main fault plane.

In their study, PDR calculated off-plane stresses for the semi-infinite nonsingular slip-weakening model in Figure 1 


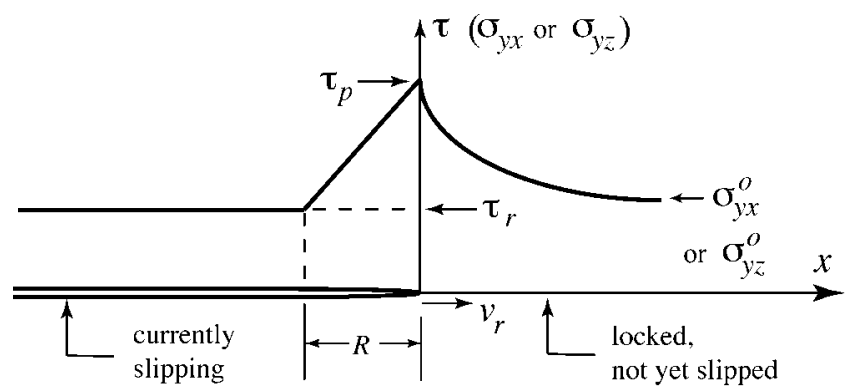

Figure 1. Nonsingular slip-weakening rupture model with a linear variation of shear stress (from peak strength $\tau_{p}$ to residual strength $\tau_{r}$ ) with distance from the crack tip in the slip-weakening zone. After Poliakov et al. (2002); semi-infinite slipping zone, corresponding to negligible stress drop $\sigma_{y x}^{o}-\tau_{r}$ (mode II) or $\sigma_{y z}^{o}-\tau_{r}$ (mode III), compared with strength drop $\tau_{p}-\tau_{r}$.

(the singular crack corresponds to a slip-weakening zone length of $R=0$ in this figure). The initial stress, or prestress, $\sigma_{i j}^{o}$ was defined such that the $y=0$ fault plane contains the intermediate principal stress axis and the slip vector, which is assumed to align with the shear traction on the plane. The rupture was assumed to propagate in the $x$ direction and two cases were considered, mode II and mode III. Mode II rupture occurs when the intermediate principal stress is in the $z$ direction and slip is in the $x$ direction, parallel to the rupture propagation. In this case, the prestress has the form:

$$
\sigma_{i j}^{o}=\left[\begin{array}{ccc}
\sigma_{x x}^{o} & \sigma_{y x}^{o} & 0 \\
\sigma_{y x}^{o} & \sigma_{y y}^{o} & 0 \\
0 & 0 & \sigma_{z z}^{o}
\end{array}\right]
$$

Mode III occurs when the intermediate principal stress is in the $x$ direction and slip is in the $z$ direction (perpendicular to the propagation). For mode III the prestress is

$$
\sigma_{i j}^{o}=\left[\begin{array}{ccc}
\sigma_{x x}^{o} & 0 & 0 \\
0 & \sigma_{y y}^{o} & \sigma_{y z}^{o} \\
0 & \sigma_{y z}^{o} & \sigma_{z z}^{o}
\end{array}\right]
$$

The origin of the $x, y$ coordinate system moves with the crack tip at velocity $v_{r}$, and for the simple steady state considered, stress and displacements fields calculated in that moving frame are time invariant.

PDR did not explicitly consider pore pressure when discussing distributions of off-fault failure predicted by their slip-weakening analysis. However, we wish to do so here and thus will regard their $\sigma_{i j}^{o}$ as Terzaghi "effective" stresses. That is, $\sigma_{i j}^{o}=\sigma_{i j}^{\text {tot, } o}+p^{o} \delta_{i j}$ where $\sigma_{i j}^{\text {tot }}$ is the total stress, so that $\sigma_{i j}^{\text {tot } o}$ denotes its initial value, and $p$ is pore pressure, with initial value $p^{0}$. We calculate the alterations $\Delta \sigma_{i j}^{\text {tot }}$ of total stress due to the propagation of the dynamic rupture in the next section. In poroelastic terminology, response should be considered to be "undrained" over the multimeter scale of interest to us here, on the short timescale during which the dynamic stress pulse traverses a material point. In such circumstances the pore pressure change, at least at points slightly off the fault plane (where other effects such as thermal pressurization of pore fluid from shear heating, inelastic dilation, and local Darcy transport may be occurring) can be calculated as:

$$
\Delta p=-B\left(\Delta \sigma_{x x}^{\mathrm{tot}}+\Delta \sigma_{y y}^{\mathrm{tot}}+\Delta \sigma_{z z}^{\mathrm{tot}}\right) / 3
$$

where $B$ is the Skempton coefficient (Rice and Cleary, 1976) for stress alteration under undrained conditions. Thus, the alterations of the effective stress are $\Delta \sigma_{i j}=\Delta \sigma_{i j}^{\text {tot }}+\Delta p \delta_{i j}$.

The plan of this article is to first show results that neglect poroelastic effects, in the sense of assuming a Skempton coefficient $B=0$, so that $\Delta p=0$ and $\Delta \sigma_{i j}=\Delta \sigma_{i j}^{\text {tot }}$. That is a case of interest in itself and allows comparison with the analogous results shown by PDR. (PDR did discuss the inclusion of $B \neq 0$ and $\Delta p \neq 0$ in their analysis of stresses favoring rupture branching near a singular crack tip, but not in their slip-weakening analysis.) Subsequently, we discuss the effects of taking $B \neq 0$ and $\Delta p \neq 0$ in the present analysis of off-fault damage; those effects can be significant.

Although the PDR slip-weakening model captures many important characteristics of the stress field near the tip of a propagating rupture, it may not be the best model for an earthquake. Because the slipping region is treated as semiinfinite (relative to the size $R$ of the slip-weakening zone), the initial stress $\sigma_{y x}^{o}$ and the residual strength $\tau_{r}$ cannot be significantly different, that is, the dynamic stress drop approaches zero in their model. PDR characterize this as a "strong but brittle" limit for faulting (meaning $\tau_{p}-\tau_{r}$ is much larger than $\sigma_{y x}^{o}-\tau_{r}$; "strong" because of the large $\tau_{p}-\tau_{r}$, "brittle" because of the small $\sigma_{y x}^{o}-\tau_{r}$ at which propagation occurs). Also, in their model, slip continues over the entire slipped zone from $0^{-}$to $-\infty$. However, seismological observations of the temporal and spatial distribution of slip during large earthquakes suggest that slip is limited to a finite zone behind the propagating crack tip. Known as the "self-healing pulse model" for earthquakes (Heaton, 1990), this is a dislocation-like model in which no slip occurs at distances $x<-L$ behind the crack tip.

In this article, we extend the work by PDR by calculating the off-plane stress field with a dislocation model in which slip is limited to a patch of length $L$ behind a crack tip that propagates at constant velocity $v_{r}$ (Fig. 2). Thus, we address a slip-weakening version of a model of a self-healing fracture, propagating with steady-state space-time dependence, as studied by Broberg (1978) and Freund (1979), and by Nielsen and Madariaga (2003) in the context of a self-similar space-time dependence. Like Broberg (1978), we consider nonuniform shear stresses along the slipping zone due to a gradual loss of strength near the fracture tip. However, we follow the Freund (1979) description of the onset of healing, as a process taking place without local alteration of stress along the slipping zone near the healing front, corresponding 
to cessation of slip without a stress singularity (Broberg [1999] later adopted the same concept for healing). Once healed, the stress necessarily rises along the newly locked portion of the fault in this class of models (Perrin et al., 1995). We thereby simulate a self-healing slip pulse (without addressing the question of why it is self-healing) and are able to consider cases where the dynamic stress drop $\sigma_{y x}^{o}-$ $\tau_{r}$ is a finite fraction of the strength drop $\tau_{p}-\tau_{r}$. Although we find that the general conclusions in PDR (summarized previously) also hold for the slip-pulse model, significant differences exist, in particular, in the spatial extent of offfault failure, as the rupture velocity approaches $c_{\mathrm{lim}}$.

\section{Stress Field Near an Elastodynamic Slip Pulse}

We follow the general approach in PDR, but in place of the semi-infinite sliding zone in Figure 1, we consider a sliding zone of finite length $L$ that moves with constant velocity $v_{r}$ in the $x$ direction as in Figure 2. The forward end of the sliding patch is at $x=0$. The shear stress on the fault plane $(y=0)$ far ahead of the crack tip is $\sigma_{y x}^{o}$ for mode II or $\sigma_{y z}^{o}$ for mode III. We use the nonsingular slip-weakening model introduced by Ida (1972) and Palmer and Rice (1973) and used in PDR. Weakening begins when shear stress on the fault $\tau$ ( $=\sigma_{y x}$ or $\sigma_{y z}$ ) first reaches a finite peak strength $\tau_{p}$ on an unslipped part of the fault. When slip $\Delta u$ begins, $\tau$ decreases with $\Delta u$, approaching $\tau_{r}$ at large $\Delta u$, somewhat as illustrated in Figure 2. We have assumed, for simplicity, a linear decrease of $\tau$ with spatial distance $x$ (that is not consistent with a linear decrease of $\tau$ with $\Delta u$ ). The peak strength $\tau_{p}=-\left(\sigma_{y y}^{o}\right) \tan \phi_{p}$ is determined by the coefficient of static friction $\left(f_{s}=\tan \phi_{p}\right)$ on the fault plane. We take $f_{s}=0.6$ corresponding to $\phi_{p} \approx 31^{\circ}$, a typical value for rock. The residual strength $\tau_{r}=-\left(\sigma_{y y}^{o}\right) \tan \phi_{r}$ is determined by the dynamic coefficient of friction $f_{d}=\tan \phi_{r}$. Since we do not know what to take for $f_{d}$ (or even if it is constant during an earthquake) we show some results for a range of $f_{d} / f_{s}=$ $\tau_{r} / \tau_{p}$ from 0.2 to 0.8 , but favor the lower value to allow laboratory-like $f_{s}$ at the onset of slip to be consistent with low frictional dissipation in large earthquake slips.

Following the reasoning in PDR, we assume that $\tau$ decreases linearly with $x$ from $\tau_{p}$ to $\tau_{r}$ over an as-yet-unknown slip-weakening zone length $R$ along the $x$ axis, writing

$$
\tau= \begin{cases}\tau_{r}+\left(1+\frac{x}{R}\right)\left(\tau_{p}-\tau_{r}\right), & \text { for }-R<x<0 \\ \tau_{r} & \text { for }-L<x<-R,\end{cases}
$$

which implicitly assumes $R<L$ (which will be seen later to be valid for a dynamic stress drop that is less than half of the strength drop. See Fig. 3).

The effective stress field in the vicinity of such a crack may be written $\sigma_{i j}=\sigma_{i j}^{o}+\Delta \sigma_{i j}$. The task is to determine $\Delta \sigma_{i j}$, vanishing at $\infty$, such that $\sigma_{y x}$ (for mode II) or $\sigma_{y z}$ (for

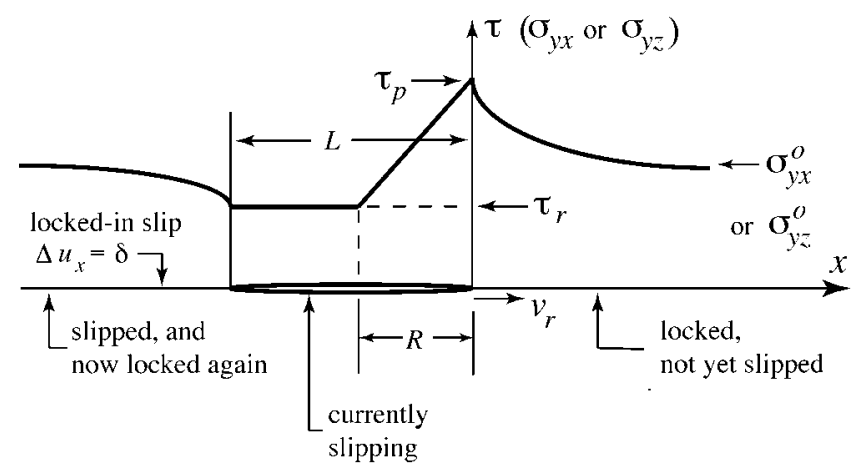

Figure 2. Nonsingular slip-weakening slip-pulse model. As in Figure 1, a linear variation of shear stress is assumed in the slip-weakening zone. The PDR model (Fig. 1) coresponds to normalizing the stress fluctuation $\Delta \sigma_{i j}$ with $\tau_{p}-\tau_{r}$ and then taking the limits $L / R \rightarrow \infty$ and $\left(\sigma_{y x}^{o}-\tau_{r}\right) /\left(\tau_{p}-\tau_{r}\right) \rightarrow 0$, such that $x / R$ and $y / R$ remain finite. In comparison, the singular crack model corresponds to first normalizing the $\Delta \sigma_{i j}$ with $\left(\sigma_{y x}^{o}-\tau_{r}\right)$ and then letting $R / L \rightarrow 0$ and $\left(\tau_{p}-\tau_{r}\right) /\left(\sigma_{y x}^{o}-\tau_{r}\right) \rightarrow \infty$ with $x / L$ and $y / L$ remaining finite. The PDR model may be regarded as providing a magnified view of the region of large but actually finite stress concentration represented by the singularity in the latter model.

$$
\left(\sigma_{y x}^{o}-\tau_{r}\right) /\left(\tau_{p}-\tau_{r}\right)
$$

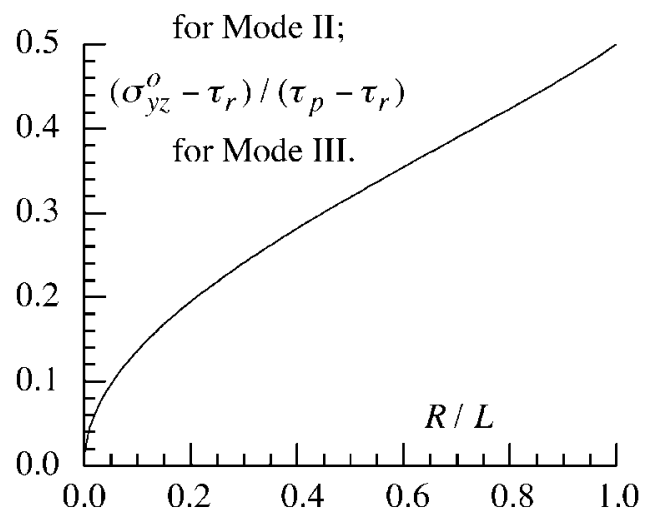

Figure 3. The scaled dynamic stress drop $\left(\left(\sigma_{y x}^{o}-\right.\right.$ $\left.\tau_{r}\right) /\left(\tau_{p}-\tau_{r}\right)$ for mode II or $\left(\sigma_{y z}^{o}-\tau_{r}\right) /\left(\tau_{p}-\tau_{r}\right)$ for mode III) as a function of $R / L$ calculated using equation (8) in the text. Note that the dynamic stress drop is 0 when $R / L=0$, corresponding to the semi-infinite crack modeled by Poliakov et al. (2002). The stress drop increases monotonically to $\left(\tau_{p}-\tau_{r}\right) / 2$ as $R / L$ $\rightarrow 1$.

mode III) has the prescribed values on the slipping patch $-L<x<0$ given by equation (2), and such that there is a smooth closure without a stress singularity at the trailing edge (Fig. 2).

PDR show that the $\Delta \sigma_{i j}^{\text {tot }}$ can be written in terms of an analytic function $M(z)$ of a complex variable $z$ (such use for $z$ is not to be confused with use of the same symbol to denote the third Cartesian coordinate) as follows: 
For mode II:

$$
\begin{gathered}
\Delta \sigma_{x x}^{\mathrm{tot}}=\Delta \sigma_{x x}-\Delta p=2 \alpha_{s} \operatorname{Im}\left[\left(1-\alpha_{s}^{2}+2 \alpha_{d}^{2}\right)\right. \\
\left.M\left(z_{d}\right)-\left(1+\alpha_{s}^{2}\right) M\left(z_{s}\right)\right] / D \\
\Delta \sigma_{y y}^{\mathrm{tot}}=\Delta \sigma_{y y}-\Delta p=-2 \alpha_{s}\left(1+\alpha_{s}^{2}\right) \\
\operatorname{Im}\left[M\left(z_{d}\right)-M\left(z_{s}\right)\right] / D, \\
\Delta \sigma_{y x}^{\mathrm{tot}}=\Delta \sigma_{y x}=\operatorname{Re}\left[4 \alpha_{s} \alpha_{d} M\left(z_{d}\right)-\left(1+\alpha_{s}^{2}\right)^{2} M\left(z_{s}\right)\right] / D .
\end{gathered}
$$

For mode III:

$$
\Delta \sigma_{y z}=\operatorname{Re}\left[M\left(z_{s}\right)\right], \quad \Delta \sigma_{x z}=\operatorname{Im}\left[M\left(z_{s}\right) / \alpha_{s}\right] .
$$

In these expressions the complex variables $z_{d}$ and $z_{s}$ are defined as $z_{d}=x+i \alpha_{d} y$ and $z_{s}=x+i \alpha_{s} y$, where $\alpha_{d}=$ $\sqrt{1-v_{r}^{2} / c_{d}^{2}}, \alpha_{s}=\sqrt{1-v_{r}^{2} / c_{s}^{2}}$, and $c_{d}$ and $c_{s}$ are the dilatational and shear-wave speeds, and "Re" and "Im" mean the real and imaginary parts. The Rayleigh function $D$ is $D=4 \alpha_{s} \alpha_{d}-\left(1+\alpha_{s}^{2}\right)^{2}$ and vanishes when $v_{r}=c_{R}$. (The expression for $\Delta \sigma_{y x}$ in equation 3 corrects a misprint in the appendix of PDR. The signs of the right sides of the expressions for $\Delta \sigma_{y x}$ in their equations $\mathrm{A} 2$ and $\mathrm{A} 3$ should be reversed, although the correct form of those equations was used in the results presented in PDR.)

For the plane strain conditions assumed in mode II, $\Delta \sigma_{z z}^{\text {tot }}=v\left(\Delta \sigma_{x x}^{\text {tot }}+\Delta \sigma_{y y}^{\text {tot }}\right)$, where $v$ is the Poisson ratio (the undrained Poisson ratio in poroelastic terminology) and thus, using (1), we calculate $\Delta p$ in equation (3) as

$$
\Delta p=-(1+v) B\left(\Delta \sigma_{x x}^{\mathrm{tot}}+\Delta \sigma_{y y}^{\mathrm{tot}}\right) / 3
$$

We always adopt $v=1 / 4$ here, consistent with $c_{d}=\sqrt{3} c_{s}$ and $c_{R} \approx 0.919 c_{s}$.

Introducing the notation $\theta^{\prime}=2 \sin ^{-1}(\sqrt{R / L})$, which varies from 0 to $\pi$ as $R / L$ varies from 0 to 1 , and letting $z$ be a proxy for either of $z_{d}$ or $z_{s}$, the appropriate analytic function $M(z)$ is shown in the Appendix to be $M(z)=M^{0}+M^{1}(z)$, where

$$
\begin{array}{r}
M^{0}=-\left(\tau_{p}-\tau_{r}\right)\left[\frac{\theta^{\prime}}{\pi}-\frac{\theta^{\prime}-\sin \theta^{\prime}}{2 \pi \sin ^{2}\left(\theta^{\prime} / 2\right)}\right], \\
M^{1}(z)=-\frac{1}{\pi}\left(\tau_{p}-\tau_{r}\right)\left\{\left(1+\frac{z}{R}\right)\left[\frac{1}{i} \ln (F(z))\right]\right. \\
\left.+\frac{z^{1 / 2}(z+L)^{1 / 2} \theta^{\prime}}{R}\right\},
\end{array}
$$

and

$$
\begin{aligned}
& F(z)=\left(\frac{\left(e^{i \theta^{\prime}}-1\right) L / 2-z-z^{1 / 2}(z+L)^{1 / 2}}{\left(e^{i \theta^{\prime}}-1\right) L / 2-z+z^{1 / 2}(z+L)^{1 / 2}}\right) \\
&\left(\frac{z-z^{1 / 2}(z+L)^{1 / 2}}{z+z^{1 / 2}(z+L)^{1 / 2}}\right) .
\end{aligned}
$$

Here, the branch cut of $z^{1 / 2}(z+L)^{1 / 2}$ is along the slipping zone such that the product approaches $z$ for $|z| \gg L$. Also, as will be clear from (8) to follow, $M^{0}=-M^{1}(\infty)$ corresponds to $\tau_{r}-\sigma_{y x}^{o}$ in mode II and to $\tau_{r}-\sigma_{y z}^{o}$ in mode III.

\section{Nondimensional Parameters of the Model}

If we make all stress alterations $\Delta \sigma_{i j}$ nondimensional by dividing by the peak strength $\tau_{p}\left(=-f_{s} \sigma_{y y}^{o}\right)$, and coordinates nondimensional in the form $x / R$ and $y / R$, the expressions for the stress alterations then depend on four nondimensional parameters for mode II. We choose these to be the scaled rupture speed $v_{r} / c_{s}$, the ratio of residual to peak strength $\tau_{r} / \tau_{p}$, the length of the slip-weakening zone relative to the length of the slip pulse $R / L$, and the Poisson ratio $v$ (which is irrelevant for mode III and which we always choose as $v=0.25$, so that $c_{d}=\sqrt{3} c_{s}$ ). The Skempton factor $B$ becomes a fifth parameter in our models considering pore pressure and undrained poroelastic response, in which $v=0.25$ is then interpreted as the undrained Poisson ratio. The ratio $\sigma_{y x}^{o} / \tau_{p}$ is implied by that parameter set (in fact, just by $\tau_{r} / \tau_{p}$ and $R / L$; see equation 8 ), but to fully represent the in-plane components of $\sigma_{i j}^{o} / \tau_{p}$, to determine zones where the predicted stresses violate the Mohr-Coulomb failure condition, we must specify two more parameters. We choose these as the ratio of the normal prestresses $\sigma_{x x}^{o} / \sigma_{y y}^{o}$, and the coefficient of static friction $f_{s}=\tau_{p} /\left(-\sigma_{y y}^{o}\right)$. The latter, which is also the only parameter needed to specify the Mohr-Coulomb failure condition, is chosen as $f_{s}=0.6$ in all the results shown here.

The dynamic stress drop, $\sigma_{y x}^{o}-\tau_{r}$ for mode II, may be expressed in scaled form as a function of $R / L$ (see equation A14 in the Appendix) as

$$
\frac{\left(\sigma_{y x}^{o}-\tau_{r}\right)}{\left(\tau_{p}-\tau_{r}\right)}=\frac{\theta^{\prime}}{\pi}-\frac{\theta^{\prime}-\sin \theta^{\prime}}{2 \pi \sin ^{2}\left(\theta^{\prime} / 2\right)}
$$

where, again, $\theta^{\prime}=2 \sin ^{-1} \sqrt{R / L}$. (This stress ratio corresponds to $1 /\left(1+S_{A}\right)$, where $S_{A}=\left(\tau_{p}-\sigma_{y x}^{o}\right) /\left(\sigma_{y x}^{o}-\tau_{r}\right)$ is the Andrews [1976b] ratio.) The same expression as in equation (8) applies for $\sigma_{y z}^{o}-\tau_{r}$ in mode III. Remarkably, rupture speed $v_{r}$ does not enter this expression; both $R$ and $L$ vary in the same way with $v_{r}$ and both vanish as $v_{r} \rightarrow c_{\text {lim }}$, as explained following. The methods of the Appendix can be used to show that such results are not limited to the particular slip-weakening law associated with the linear-in- $x$ stress distribution adopted here; for all slip-weakening laws, the 2D steady slip-pulse solution has the features that $R / L$ is a function only of the scaled stress drop (but generally different in form from equation 8), independently of $v_{r}$, and that both $R$ and $L$ vanish as $v_{r} \rightarrow c_{\mathrm{lim}}$. Equation (8) is plotted as in Figure 3 . Thus, choosing a particular $R / L$ is equivalent to choosing the scaled dynamic stress drop (which may then be regarded as a prescribed parameter in place of $R / L$ ) or, thinking of $\tau_{p}$ and $\tau_{r}$ as given material properties, to choosing the initial shear stress $\sigma_{y x}^{o}$. Note that the dynamic stress drop $\sigma_{y x}^{o}-\tau_{r}$ is less than $1 / 2$ the strength drop for all $R<L$ (i.e., for all 
$\left.\theta^{\prime}<\pi\right)$, and that $\left(\sigma_{y x}^{o}-\tau_{r}\right) /\left(\tau_{p}-\tau_{r}\right) \rightarrow 0$ as $R / L \rightarrow 0$ $\left(\theta^{\prime} \rightarrow 0\right)$, corresponding to the semi-infinite crack modeled by PDR.

The size of the slip-weakening zone, $R$, depends on the fracture energy $G$, the shear modulus $\mu$, the strength drop $\left(\tau_{p}-\tau_{r}\right)$, the scaled rupture velocity $v_{r} / c_{s}$, and the dynamic stress drop (which we parameterize using $\theta^{\prime}=2 \sin ^{-1}$ $\sqrt{R / L}$ as previously). In this slip-weakening context, $G$ is defined by $G=\int\left[\tau(\Delta u)-\tau_{r}\right] d(\Delta u)$ (Palmer and Rice, 1973; Rice, 1980), where $\tau(\Delta u)$ is the slip-weakening function implied through the elasticity solution for our assumed linear-in- $x$ stress distribution in Figure 2 and the integral extends to sufficiently large slips $\Delta u$ that $\tau(\Delta u)$ becomes coincident with $\tau_{r}$. We show in the Appendix, in equations (A25) and (A26), that

$$
R=\frac{\mu G}{\left(\tau_{p}-\tau_{r}\right)^{2}} \frac{F\left(v_{r}\right)}{h\left(\theta^{\prime}\right)},
$$

where

$$
h\left(\theta^{\prime}\right)=\left(\frac{\theta^{\prime}}{\pi}-\frac{\theta^{\prime}-\sin \theta^{\prime}}{2 \pi \sin ^{2}\left(\theta^{\prime} / 2\right)}\right)\left(\frac{\theta^{\prime}-\sin \theta^{\prime} \cos \theta^{\prime}}{4 \sin ^{4}\left(\theta^{\prime} / 2\right)}\right),
$$

which varies only modestly, from $h\left(0^{+}\right)=16 / 9 \pi \approx 0.566$ to $h(\pi)=\pi / 8 \approx 0.393$, and

$$
F\left(v_{r}\right)=\left\{\begin{array}{l}
D /\left[\alpha_{s}\left(1-\alpha_{s}^{2}\right)\right] \text { for mode II } \\
\alpha_{s} \text { for mode III. }
\end{array}\right.
$$

Here, $D=4 \alpha_{s} \alpha_{d}-\left(1+\alpha_{s}^{2}\right)^{2}$ is the Rayleigh factor defined previously. The functions $F\left(v_{r}\right)$ diminish with rupture speed and vanish when $v_{r} \rightarrow c_{\text {lim }}$, with the following limits:

$$
F\left(v_{r}\right)=\left\{\begin{array}{l}
1 /(1-v) \text { when } v_{r}=0 \\
0 \text { when } v_{r}=c_{R} \\
1 \text { when } v_{r}=0 \\
0 \text { when } v_{r}=c_{s}
\end{array}\right\} \text { for mode II }
$$

where $v$ is the Poisson ratio. The ratio $F\left(v_{r}\right) / F(0)$ corresponds to what is called $1 / f_{\text {II }}\left(v_{r}\right)$ for mode II, and $1 / f_{\text {III }}\left(v_{r}\right)$ for mode III, in Rice (1980) and PDR.

We write the value of $R$ at low speed $\left(v_{r}=0^{+}\right)$, but at the same fixed $R / L$ (or fixed stress drop), as $R_{o}$ where $R_{o}=$ $\left[\mu G /\left(\tau_{p}-\tau_{r}\right)^{2}\right] F(0) / h\left(\theta^{\prime}\right)$. Hence,

$$
\frac{R}{R_{o}}=\frac{F\left(v_{r}\right)}{F(0)}=\frac{(1-v) D}{\alpha_{s}\left(1-\alpha_{s}^{2}\right)}
$$

for mode II, and $R / R_{o}=\alpha_{s}$ for mode III. Thus $R / R_{o}$ is a function of $v_{r}$ only. A slipping length $L_{o}$ may be similarly defined, just by equating $R_{o} / L_{o}$ to the fixed $R / L$. Then it follows that $L / L_{o}=R / R_{0}$, so that $L / L_{o}$ is the same function of $v_{r}$ as in equation (12).

Because $R_{o}$ depends on $R / L$, we choose to normalize all lengths in the problem by the value of $R_{o}$ when $L / R \rightarrow \infty$ (that is, when the dynamic stress drop is a negligible fraction of the strength drop), which we call $R_{o}^{*}$. This is obviously invariant to $v_{r}$ and to the magnitude of the stress drop. Because $\lim h\left(\theta^{\prime}\right)=h\left(0^{+}\right)=16 / 9 \pi$, we have

$$
R_{o}^{*}=\frac{9 \pi F(0)}{16} \frac{\mu G}{\left(\tau_{p}-\tau_{r}\right)^{2}}=\frac{9 \pi}{16(1-v)} \frac{\mu G}{\left(\tau_{p}-\tau_{r}\right)^{2}},
$$

for mode II, exactly as in PDR, and the $(1-v)$ is deleted for mode III. Hence, using equations (9) and (13), we scale $R$ for mode II as

$$
\frac{R}{R_{o}^{*}}=\frac{16(1-v)}{9 \pi} \frac{F\left(v_{r}\right)}{h\left(\theta^{\prime}\right)}
$$

and $L$ as $L / R_{o}^{*}=(L / R)\left(R / R_{o}^{*}\right)$, where $L / R$ is one of the given parameters. For mode III, the $(1-v)$ is again deleted. (Note that our $R_{o}^{*}$ coincides with what PDR called $R_{o}$, because they dealt only with the $\theta^{\prime}=0$ case of vanishing scaled stress drop.)

As in PDR, $R$ decreases to zero as $v_{r}$ increases to its limiting value $c_{\text {lim. }}$. Because $L / R$ is fixed for a given, scaled, stress drop by equation (8), $L$ also approaches zero. However, the final slip displacement $\delta$ that is locked-in on healing is independent of $v_{r}$, so that our slip-pulse solution approaches that of a step Volterra dislocation moving at speed $c_{\text {lim }}$. That is because, as we show in equation (A23),

$$
\delta=\frac{G}{\sigma_{y x}^{o}-\tau_{r}}=\frac{16(1-v)}{9 \pi} \frac{\left(\tau_{p}-\tau_{r}\right)^{2}}{\left(\sigma_{y x}^{o}-\tau_{r}\right) \mu} R_{o}^{*},
$$

for mode II, where the latter form uses (13) also. Thus, the locked-in displacement depends only on the fracture energy and dynamic stress drop but is independent of the velocity. This result (15) also holds for mode III, which differs only by the change of stress drop to $\sigma_{y z}^{o}-\tau_{r}$ and deletion of the $(1-v)$ in the latter form. The result, rewritten as $\sigma_{y x}^{o} \delta=$ $\tau_{r} \delta+G$, is easy to interpret. It says that the work $\sigma_{y x}^{o} \delta$ of the remote stress field in advance of the rupture front over a unit area is balanced by the total dissipation, which is the sum of $\tau_{r} \delta$ in friction dissipation at the residual level, plus $G$ by stresses $\tau(\Delta u)-\tau_{r}$ in excess of residual.

As suggested, there is a certain slip-weakening relation $\tau=\tau(\Delta u)$ implied by our analysis, which we have simplified by assuming a linear variation of stress with distance as in Figure 2, corresponding to a specified fracture energy $G$, which is interpretable as $G=\int\left[\tau(\Delta u)-\tau_{r}\right] d(\Delta u)$. The resulting weakening function $\tau(\Delta u)$ was plotted by Palmer and Rice (1973) for the $R / L=0$ limit and $v_{r}=0^{+}$case that they considered. Rice (1980) showed, again for the $R / L=$ 0 limit, that for dynamic propagation, the function $\tau(\Delta u)$ implied by this procedure is independent of $v_{r}$. A similar result holds here: For a given scaled dynamic stress drop, 
hence given $R / L$ and given fracture energy $G$, the implied slip-weakening function is independent of $v_{r}$. However, in our present work, the precise form of that implied $\tau(\Delta u)$ depends on $R / L$ (although it starts at the same $\tau_{p}$, finishes at the same $\tau_{r}$ and has the same integral $G$ for all $R / L$ ). We show in the Appendix how to determine $\tau(\Delta u)$ and, by comparing results for the limit cases $R / L=0$ and $R / L=1$ (see the final figure of the Appendix), we demonstrate that the dependence on $R / L$ is very weak, suggesting therefore that it may be safely neglected.

\section{Off-Plane Coulomb Failure Induced by a Slip Pulse: Mode II}

The potential for bending or forking of the slip-pulse onto planes intersecting a singular crack tip is exactly as calculated by PDR for the semi-infinite crack, because the stress field in the immediate vicinity of the tip is the same in both cases. However, the spatial extent of Coulomb failure on slip surfaces removed from the crack tip can be quite different for the two cases. Slip will occur when the Coulomb stress on a plane, defined as $\tau+f_{s} \sigma_{n}$, is greater than 0 . (Recall that $\sigma_{n}$ is negative for compression and, considering pore pressure, the stresses are effective stresses.) We will produce figures similar to figures 11 and 13 in PDR to effect a direct comparison between the mode II finite slip pulse modeled here and the mode II semi-infinite crack modeled by PDR. Those figures in PDR explore the effects of parameters $\sigma_{x x}^{o} / \sigma_{y y}^{o}, v_{r} / c_{s}$, and $\tau_{r} / \tau_{p}$ on the spatial distribution of Coulomb failure. We use the same range of parameters as in figures 11 and 13 in PDR for a range of the new parameter $R / L=0.001,0.1,0.5$, and 0.9 . The figures in PDR correspond to the case $R / L=0$. We explore mode III in the next section.

The new figures were calculated as follows: All stresses were scaled by $\tau_{p}$. The diagonal elements of the remote stress field are then $\sigma_{y y}^{o} / \tau_{p}=-1 / f_{s}$, where we take $f_{s}=0.6$ for the static coefficient of friction, and $\sigma_{x x}^{o} / \tau_{p}=\left(\sigma_{x x}^{o} / \sigma_{y y}^{o}\right) \sigma_{y y}^{o} /$ $\tau_{p}$, where the stress ratio is a given parameter. The remote shear stress is given by (A14) in the Appendix which, scaled by $\tau_{p}$, gives

$$
\frac{\sigma_{y x}^{o}}{\tau_{p}}=\left(\frac{\theta^{\prime}}{\pi}-\frac{\theta^{\prime}-\sin \theta^{\prime}}{2 \pi \sin ^{2}\left(\theta^{\prime} / 2\right)}\right)\left(1-\frac{\tau_{r}}{\tau_{p}}\right)+\frac{\tau_{r}}{\tau_{p}},
$$

where $\tau_{r} / \tau_{p}$ and $\theta^{\prime}=2 \sin ^{-1} \sqrt{R / L}$ are given parameters.

The changes in effective stress due to the cut, $\Delta \sigma_{i j}$, were calculated using equations (3), (5), (6), and (7), with $B=0$ in (5) so that $\Delta p=0$ and $\Delta \sigma_{i j}=\Delta \sigma_{i j}^{\text {tot }}$ (Figs. 5 to 10 ), and subsequently with $B \neq 0$ (Figs. 11 and 12). Note that the stresses in these equations have the factor $\left(\tau_{p}-\tau_{r}\right)$ which, because we are scaling all stresses by $\tau_{p}$, becomes $\left(1-\tau_{r} / \tau_{p}\right)$. The lengths $z, R$, and $L$ in these equations were scaled by $R_{o}^{*}$ as discussed previously. The stress components were calculated as $\sigma_{i j}=\sigma_{i j}^{o}+\Delta \sigma_{i j}$ on a grid of points $-2<x / R_{o}^{*}$

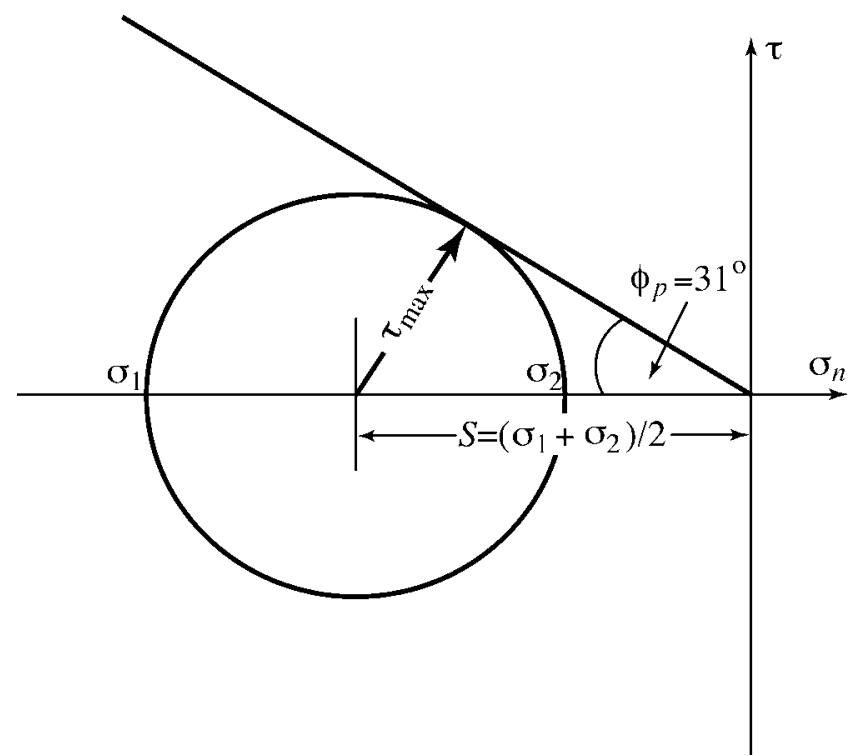

Figure 4. Mohr circle in terms of effective stress, illustrating the condition for Coulomb failure on the most favorably oriented plane.

$<2,-2<y / R_{o}^{*}<2$. At each grid point, we asked whether frictional sliding had occurred on the most favorably oriented plane. This is most easily visualized using the Mohr circle diagram in Figure 4 for which the principal stresses are $\sigma_{1}=S-\tau_{\max }$ and $\sigma_{2}=S+\tau_{\max }$, where $S=(1 / 2)\left(\sigma_{x x}\right.$ $\left.+\sigma_{y y}\right)$ and $\tau_{\max }=(1 / 2)\left[\left(\sigma_{x x}-\sigma_{y y}\right)^{2}+4 \sigma_{y x}^{2}\right]^{1 / 2}$. The angle $\psi$ between the $\sigma_{1}$ and the $x$ axis is $\psi=(1 / 2) \tan ^{-1}\left[2 \sigma_{y x} /\right.$ $\left.\left(\sigma_{x x}-\sigma_{y y}\right)\right]$.

Referring to the Mohr circle in terms of effective stress in Figure 4, slip first occurs when the circle touches the friction line $\tau=-\sigma_{n} \tan \phi_{p}$. This occurs when $\tau_{\max }=-S$ $\sin \phi_{p}$. Hence, for any stress state where $\tau_{\max } \geq-S \sin \phi_{p}$, slip will have occurred on the most favorable plane, and possibly others. We defined $\tau_{\text {Coulomb }}=-S \sin \phi_{p}$ and, at each grid point, computed the ratio $\tau_{\max } / \tau_{\text {Coulomb. Slip will }}$ have occurred at any point where $\tau_{\max } / \tau_{\text {Coulomb }} \geq 1$. The critical planes on which slip first occurs make angle of $\pm(1 / 2)\left(\pi / 2-\phi_{p}\right)$ with respect to $\sigma_{1}$. For $\phi_{p}=31^{\circ}$, the critical planes are oriented at angles $\psi \pm 29.5^{\circ}$ relative to the $x$ axis. These planes are indicated as line segments on the contour plots of $\tau_{\max } / \tau_{\text {Coulomb. }}$. We also check to see if the least principal stress may have turned positive, that is, tensile.

Effect of Rupture Velocity, Slip-Weakening Zone Size, and the Orientation of the Initial Stress Field

The effects of rupture velocity $v_{r} / c_{s}$, the slip-weakening zone size $R / L$, and the initial stress ratio $\sigma_{x x}^{o} / \sigma_{y y}^{o}$ on the offplane stress field are explored in Figures 5 through 9 for the case $B=0$. These figures are formatted as in PDR where the ratio $\tau_{\max } / \tau_{\text {Coulomb }}$ is contoured, and areas where this ratio is greater than 1 are lightly shaded. Within this lightly 


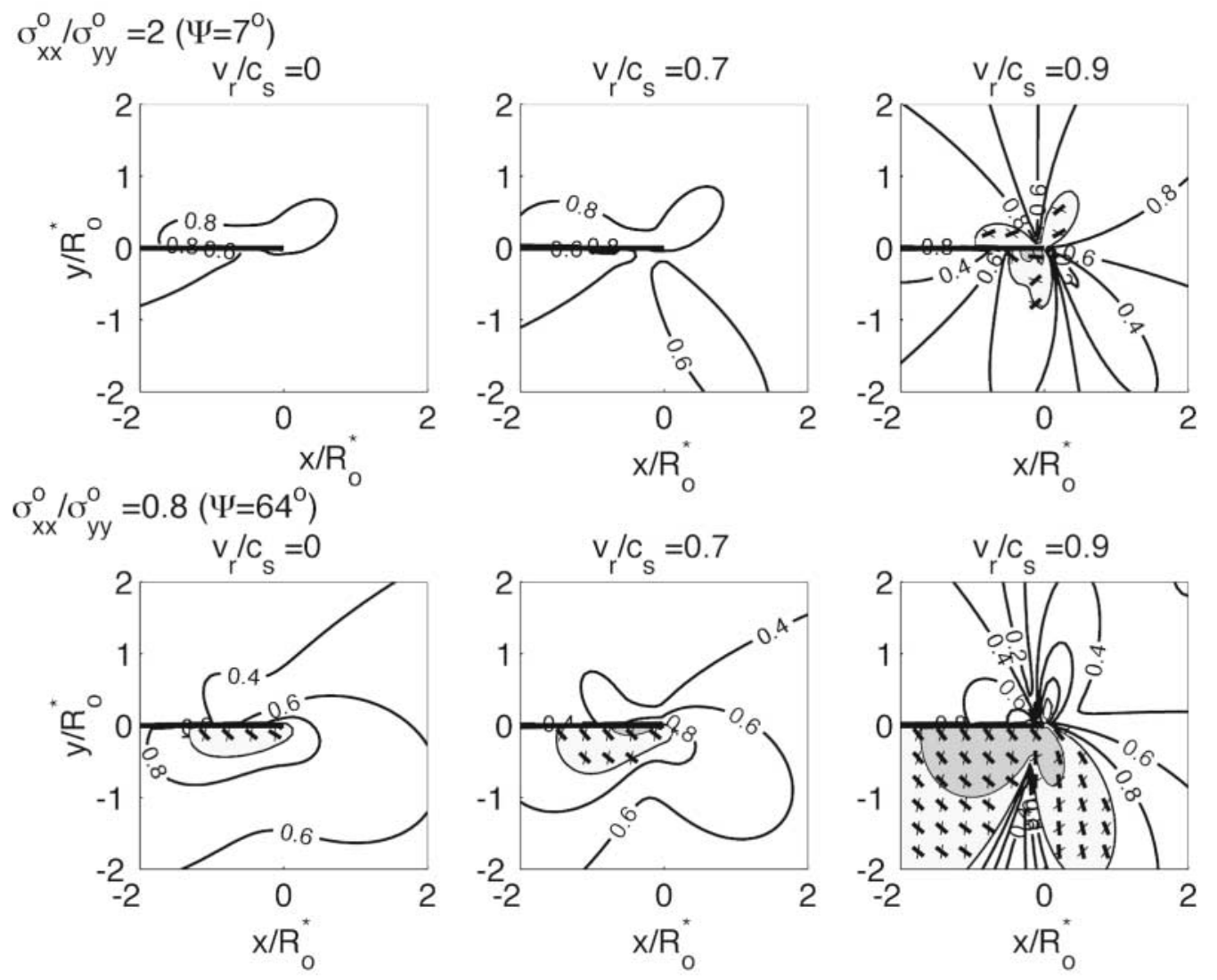

Figure 5. Contour plot of $\tau_{\max } / \tau_{\text {Coulomb }}$ as a function of position surrounding the tip of a propagating slip pulse when the weakening zone length is very small compared with the slipping length of the pulse $(R / L=0.001$, meaning that the scaled stress drop is 0.013$)$. Within the shaded areas $\tau_{\max } / \sigma_{\text {Coulomb }}>1$ and slip on pre-existing fractures is possible. The short heavy lines within the shaded areas indicate the orientation of optimal planes for right-lateral slip, the short lighter lines for left-lateral slip. In the darker shaded region, a principal stress is predicted to be tensile. The three columns of plots are for different values of the scaled propagation velocity $v_{r} / c_{s}=0,0.7$, and 0.9. In the top row of plots $\sigma_{x x}^{o} / \sigma_{y y}^{o}=2\left(\psi=7^{\circ}\right)$, in the bottom row $\sigma_{x x}^{o} / \sigma_{y y}^{o}=0.8$ $\left(\psi=64^{\circ}\right)$. All calculations in this figure assume $\tau_{r} / \tau_{p}=0.2$. Poroelastic coupling is neglected, $B=0$. This figure is identical with figure 11 in Poliakov et al. (2002) for which $L \rightarrow \infty$ and $R / L \rightarrow 0$.

shaded region, Coulomb slip is possible and the optimal orientations of slip planes are indicated by line segments. The heavier-weight lines are for right-lateral slip and the lighter are for left-lateral slip (where we have modeled a right-lateral rupture on the main fault plane). In some cases the minimum compressive effective stress close to the rupture becomes tensile (positive in Fig. 4). We indicate such regions with darker shading. Within tensile regions extensive fragmentation probably occurs.

In each figure, the upper row of three panels is calculated assuming a ratio of initial stresses $\sigma_{x x}^{o} / \sigma_{y y}^{o}=2$ (maximum compression direction at a low angle to the fault plane), whereas in the lower three panels this ratio is 0.8 and the compression direction is at a high angle to the fault plane. In each case we give the angle $\Psi=(1 / 2) \tan ^{-1}\left[2 \sigma_{y x}^{o} /\right.$ $\left(\sigma_{x x}^{o}-\sigma_{y y}^{o}\right)$ ] between the maximum compression direction and the fault plane. The residual stress ratio in each case is taken to be $\tau_{r} / \tau_{p}=0.2$.
In Figure 5, the weakening zone length is assumed to be very small in comparison with the length of the slip pulse $(R / L=0.001)$, thus approximating the semi-infinite crack modeled by PDR. As expected, this figure is nearly identical with figure 11 in the PDR article. In Figures 6, 7, and 8, R/L is progressively increased to $0.1,0.5$, and 0.9 . Note that increasing $R / L$ decreases the stress concentration at the crack tip and thus reduces the size of the shaded slip region (for the same slip velocity and initial stress field). The effect of increasing slip velocity is to increase the off-fault stresses. This is largely because $L$ decreases with slip speed (as discussed subsequently in more detail). Because we fix the ratio $R / L, R$ also decreases with increasing $v_{r} / c_{s}$, thus sharpening the stress concentration and increasing off-plane stresses.

The effect of the orientation of the initial stress is the same as that documented by PDR. When the initial maximum compression direction is at a low angle to the fault plane $\left(\sigma_{x x}^{o} / \sigma_{y y}^{o}=2\right)$, Coulomb failure occurs on both sides of the 


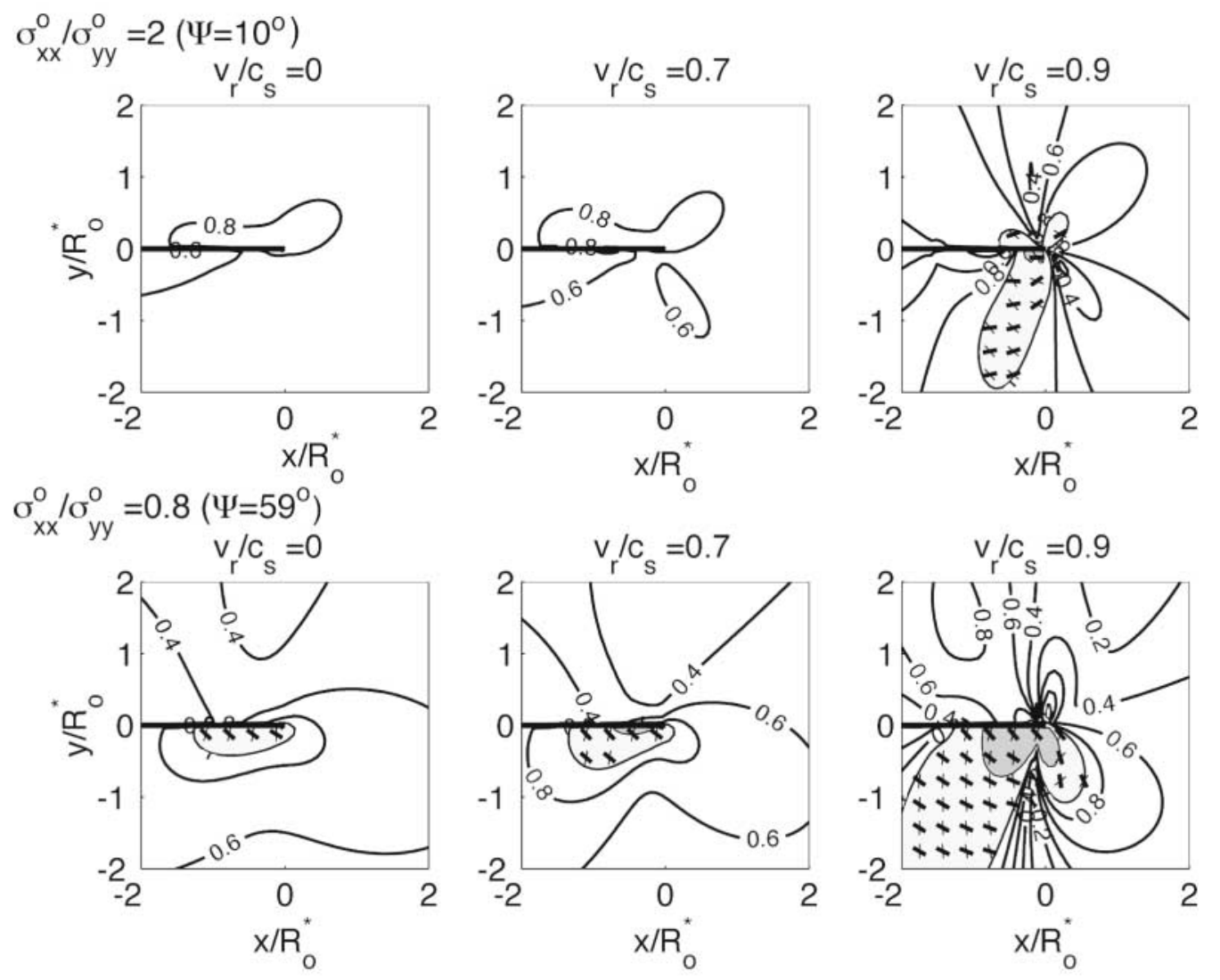

Figure 6. The same as Figure 5, except that $R / L=0.1$ (scaled stress drop $=0.136$ ). The larger weakening zone length produces less off-fault stress at a comparable slip velocity. Note that the angle $\Psi=(1 / 2) \tan ^{-1}\left[2 \sigma_{y x}^{o} /\left(\sigma_{x x}^{o}-\sigma_{y y}^{o}\right)\right]$ between the maximum precompression direction and the fault plane is slightly different for different values of $R / L$; now $\Psi=10^{\circ}$ in the top row and $59^{\circ}$ in the bottom. Like in all of Figures 5 to 10 here, $B=0$, although Figure 11 to follow examines the same cases, but with $B=0.6$.

fault plane. When it is at a higher angle $\left(\sigma_{x x}^{o} / \sigma_{y y}^{o}=0.8\right)$, failure is mostly limited to the extensional side of the fault plane. Figure 9 shows that the spatial extent of the Coulomb failure zone does not expand significantly as $v_{r} \rightarrow c_{\lim }=c_{R}$ $\approx 0.92 c_{s}$.

\section{Effect of the Residual Strength Ratio $\tau_{r} / \tau_{p}$}

Because we do not know the value of dynamic friction or the peak strength during an earthquake, we treat the ratio $f_{d} / f_{s}=\tau_{r} / \tau_{p}$ as an adjustable parameter. In the preceding section, Figures 5-9 were calculated by assuming a fairly low value of $\tau_{r} / \tau_{p}=0.2$. In Figure 10 we explore the effect of increasing $\tau_{r} / \tau_{p}$ to 0.5 and 0.8 for two values of $R / L$. As in PDR, we assume $v_{r} / c_{s}=0.9$ and $\sigma_{x x}^{o} / \sigma_{y y}^{o}=1\left(\psi=45^{\circ}\right)$ so that the top row in Figure 10 , where $R / L=0.001$, is essentially identical with figure 13 in PDR where $L / R \rightarrow \infty$. The bottom row in Figure 10 is identical with the top row except that $R / L=0.1$. Extensive Coulomb failure also occurs on the compressive side of the slip pulse when $\tau_{r} / \tau_{p}=$ 0.8. In this case the shear stresses $\sigma_{y x}$ are much larger than for $\tau_{r} / \tau_{p}=0.2$, which is presumably the cause of the effect.

\section{Effect of Poroelastic Coupling, $B \neq 0$ and $\Delta p \neq 0$}

We illustrate the effects of nonnegligible poroelastic coupling $(B \neq 0)$ in Figures 11 and 12. Figure 11 is for the same set of parameters as in Figure 6, except that fairly strong poroelastic coupling is now included with $B=0.6$. The remaining parameters are $R / L=0.1, \tau_{r} / \tau_{p}=0.2, v_{r} / c_{s}$ $=0,0.7$, and 0.9 in the three columns, and $\sigma_{x x}^{o} / \sigma_{y y}^{o}=2$ and 0.8 in the two rows. The effect of poroelastic coupling in the simple way we analyze it here (neglecting the usually modest effect on the total stress field $\left.\sigma_{i j}^{\text {tot }}\right)$ is to leave the deviatoric parts, $\left(\sigma_{x x}-\sigma_{y y}\right) / 2$ and $\sigma_{y x}$, of the effective stress field the same as when $B=0$, but to buffer the effect of the mean stress change because

$$
\Delta\left(\sigma_{x x}+\sigma_{y y}\right) / 2=[1-2(1+v) B / 3] \Delta\left(\sigma_{x x}^{\mathrm{tot}}+\sigma_{y y}^{\mathrm{tot}}\right) / 2 .
$$

The (bracketed) buffering factor is 0.5 for the case in Figure 11 with $B=0.6$. Thus, the center of the Mohr circle (Fig. 4) gets translated less than would be the case when $B=0$, but its radius is unaltered. On the compressional side of the fault, the center of the Mohr circle is translated 50\% less to 


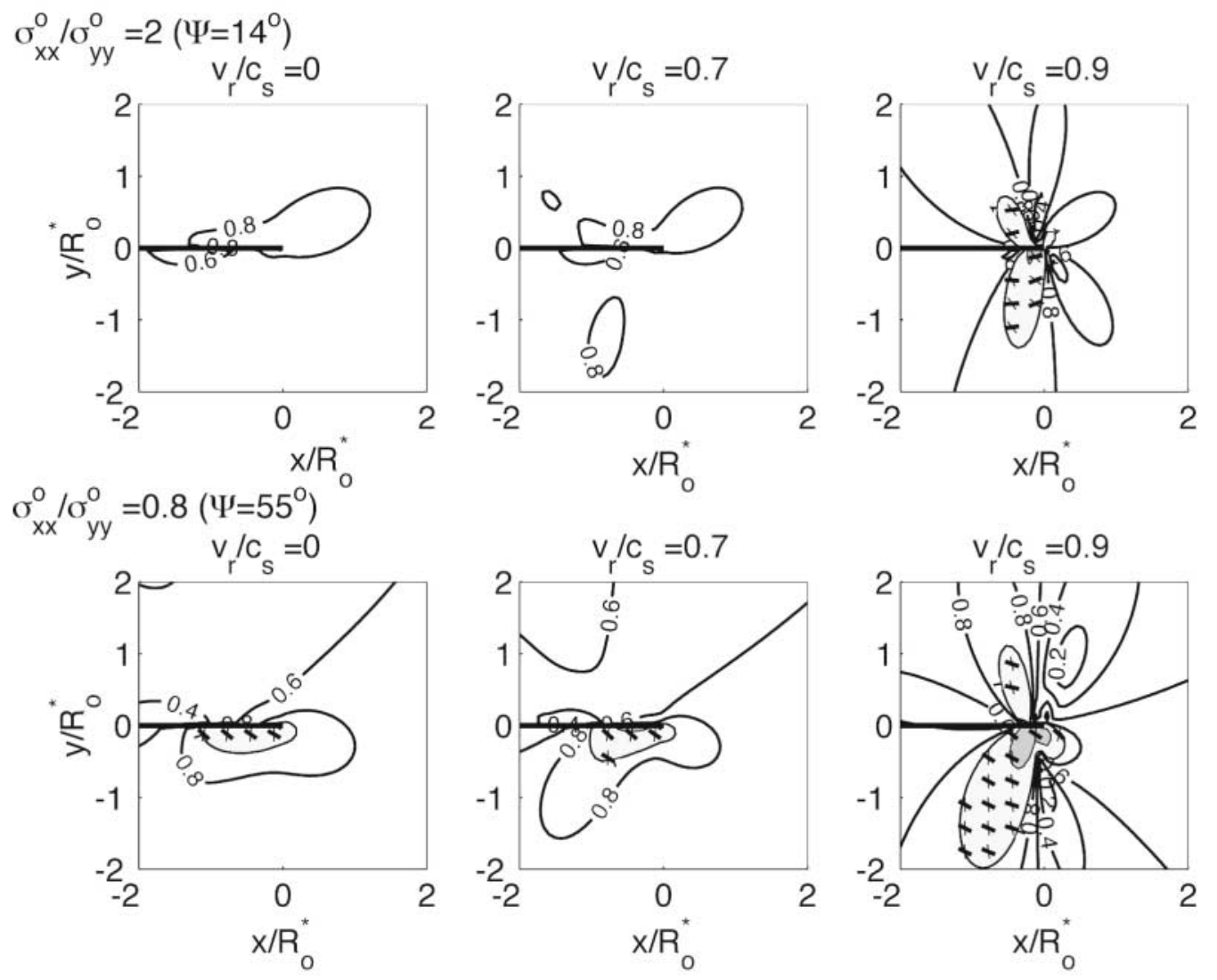

Figure 7. The same as Figures 5 and 6, except that $R / L=0.5$ (scaled stress drop $=0.318 ; \Psi=14^{\circ}$ in the top row and $55^{\circ}$ in the bottom).

the left, thereby enhancing the area of Coulomb failure. On the tensile side, it is translated $50 \%$ less to the right, thereby reducing both the area of Coulomb failure and the zone of tensile stressing. Comparison of Figure $11(B=0.6)$ with Figure $6(B=0)$ shows this effect.

Figure 12 shows the poroelastic coupling results with $B=0.3$ in the top row and $B=0.6$ in the bottom one. This is for $v_{r} / c_{s}=0.9, R / L=0.1$, and $\sigma_{x x}^{o} / \sigma_{y y}^{o}=1$ in all panels, and for strength ratios $\tau_{r} / \tau_{p}=0.2,0.5$, and 0.8 in the three columns. Those parameters other than $B$ are the same as in the bottom row of Figure 10, which thus provides the case $B=0$ for comparison. Again, the effect is to enhance failure on the compressional side and reduce it on the extensional side, although the effects are modest and almost negligible when $B=0.3$.

\section{Off-Plane Coulomb Failure Induced by a Slip Pulse: Mode III}

The antiplane shear stresses in mode III are $\sigma_{y z}=\sigma_{y z}^{o}$ $+\Delta \sigma_{y z}$, where $\Delta \sigma_{y z}$ was calculated by using equations (4), (6), and (7). As in PDR, we assumed $\sigma_{x x}^{o}=\sigma_{y y}^{o}$. Off-fault slip in this case was determined by comparing the shear stress $\tau=\sqrt{\sigma_{y z}^{2}+\sigma_{x z}^{2}}$ with $\tau_{p}$. In Figures 13 and 14, isolines of $\tau / \tau_{p}$ are plotted and areas of potential slip $\left(\tau / \tau_{p}>1\right)$ are shaded. Directions of maximum shear, $\theta_{\max }=\tan ^{-1}\left(-\sigma_{x z} /\right.$ $\left.\sigma_{y z}\right)$, are indicated by line segments. Planes of maximum Coulomb shear are not perpendicular to the plane of the figure, but intersect along the maximum shear directions shown (see PDR). Figure 13 is for the case $R / L=0.001$ and is nearly identical with Figure 15 in PDR, where $L \rightarrow \infty$. Figure 14 is for $R / L=0.5$ and shows that increasing this parameter for the slip pulse decreases the spatial extent of off-fault failure.

\section{Estimating the Fracture Energy}

Rice (2000) gave the following expression, based on the Freund (1979) solution, for the fracture energy of a singular slip pulse of length $L$ and displacement $\delta$ traveling with constant velocity $v_{r}$ :

$$
G=\frac{\mu \delta^{2}}{\pi L} F\left(v_{r}\right),
$$

where $F\left(v_{r}\right)$ is given by (11). In terms of the model of Figure 2 , that singular crack limit corresponds to first normalizing the stress fluctuation $\Delta \sigma_{i j}$ with $\left(\sigma_{y x}^{o}-\tau_{r}\right)$ and then letting $R / L \rightarrow 0$ and $\left(\tau_{p}-\tau_{r}\right) /\left(\sigma_{y x}^{o}-\tau_{r}\right) \rightarrow \infty$, but with $x / L$ and $y / L$ remaining finite. In contrast, the model addressed by PDR 

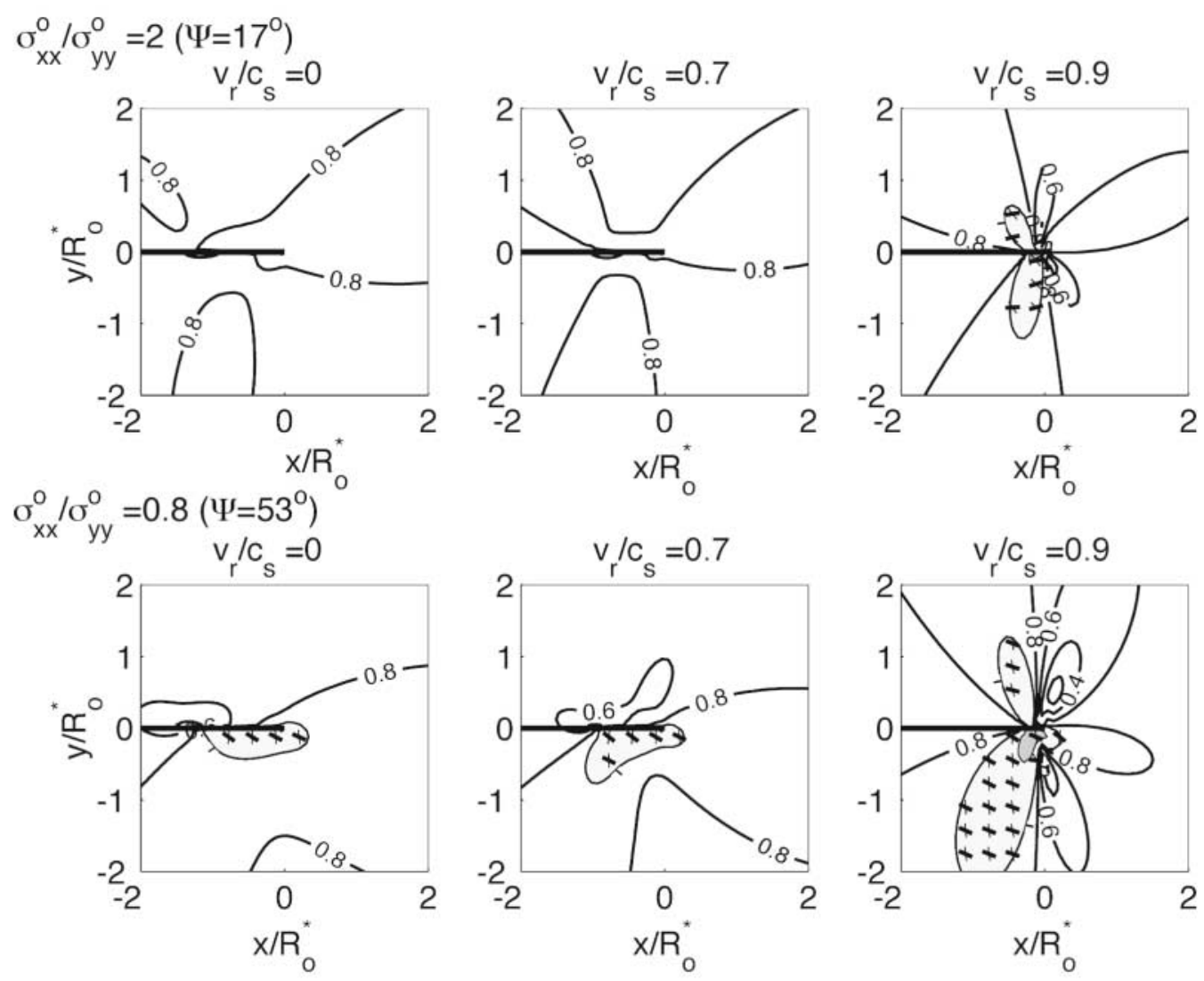

Figure 8. The same as Figures 5, 6, and 7, except that $R / L=0.9$ (scaled stress drop $=0.459 ; \Psi=17^{\circ}$ in the top row and $53^{\circ}$ in the bottom row).

(Fig. 1) corresponds to normalizing the stress fluctuation $\Delta \sigma_{i j}$ with $\tau_{p}-\tau_{r}$ and then taking the same limits as previously, now more naturally expressed as $L / R \rightarrow \infty$ and $\left(\sigma_{y x}^{o}\right.$ $\left.-\tau_{r}\right) /\left(\tau_{p}-\tau_{r}\right) \rightarrow 0$, but now such that $x / R$ and $y / R$ remain finite in the limit. The PDR model may be regarded as providing a magnified view of the region of large but actually finite stress concentration that is represented by the unbounded term of the singular crack model. Rice (2000) used Heaton's (1990) estimates of $L, \delta$, and $v_{r}$ for seven earthquakes to calculate from (17) an average value of $G \approx 2$ $\mathrm{MJ} / \mathrm{m}^{2}$.

We now repeat this analysis using our more general results for the slip-weakening slip pulse. We begin with equation (A24),

$$
G=\frac{h\left(\theta^{\prime}\right)}{F\left(v_{r}\right)} \frac{\left(\tau_{p}-\tau_{r}\right)^{2} R}{\mu},
$$

where $h\left(\theta^{\prime}\right)$ is given by equation (10) and $F\left(v_{r}\right)$ by (11). Equation (A21) can be used to write:

$$
\tau_{p}-\tau_{r}=\frac{\mu \delta}{R} F\left(v_{r}\right) \frac{4 \sin ^{4}\left(\theta^{\prime} / 2\right)}{\theta^{\prime}-\sin \theta^{\prime} \cos \theta^{\prime}} .
$$

Also, we use $R=L \sin ^{2}\left(\theta^{\prime} / 2\right)$ to change the length scale to $L$, and use (10) for $h\left(v_{r} / c_{s}, \theta^{\prime}\right)$, to get:

$$
\begin{aligned}
G^{*} \equiv \frac{G}{\left(\mu \delta^{2} / \pi L\right)}= & \left(\frac{4 \pi \sin ^{2}\left(\theta^{\prime} / 2\right)}{\theta^{\prime}-\sin \theta^{\prime} \cos \theta^{\prime}}\right) \\
& \left(\frac{\theta^{\prime}}{\pi}-\frac{\theta^{\prime}-\sin \theta^{\prime}}{2 \pi \sin ^{2}\left(\theta^{\prime} / 2\right)}\right) F\left(v_{r}\right)
\end{aligned}
$$

The product of the $\theta^{\prime}$-dependent factors in (20) varies from 1 when $\theta^{\prime} \rightarrow 0$ (i.e., when $R / L \rightarrow 0$ ), thus verifying (17) in that limit, to 2 when $\theta^{\prime}=\pi(R / L=1)$. Equation (20) is plotted in Figure 15. For $R / L=0, G^{*}$ decreases smoothly from $1 /(1-v)$ (mode II) or 1 (mode III), when $v_{r}=0^{+}$ toward 0 when $v_{r}=c_{\text {lim }}$. The effect of increasing $R / L$ is to increase $G^{*}$. The maximum increase is a factor of 2 (for both modes II and III) when $R=L$.

Whereas the scaled fracture energy $G^{*}$ decreases smoothly to 0 with increasing rupture speed, this is because it is scaled by $L$, which itself decreases to 0 . The unscaled fracture energy $G$ is independent of the rupture speed for a given, speed-independent, slip-weakening function $\tau(\Delta u)$.

\section{Interpretation of Seismically Observed Slip Pulses}

Following Rice (2000), we used the estimates of $L, \delta$, and $v_{r}$ given by Heaton (1990) from seismic slip inversions for seven events (Table 1 ) to calculate $G, \sigma_{x y}^{o}-\tau_{r}$, slip- 

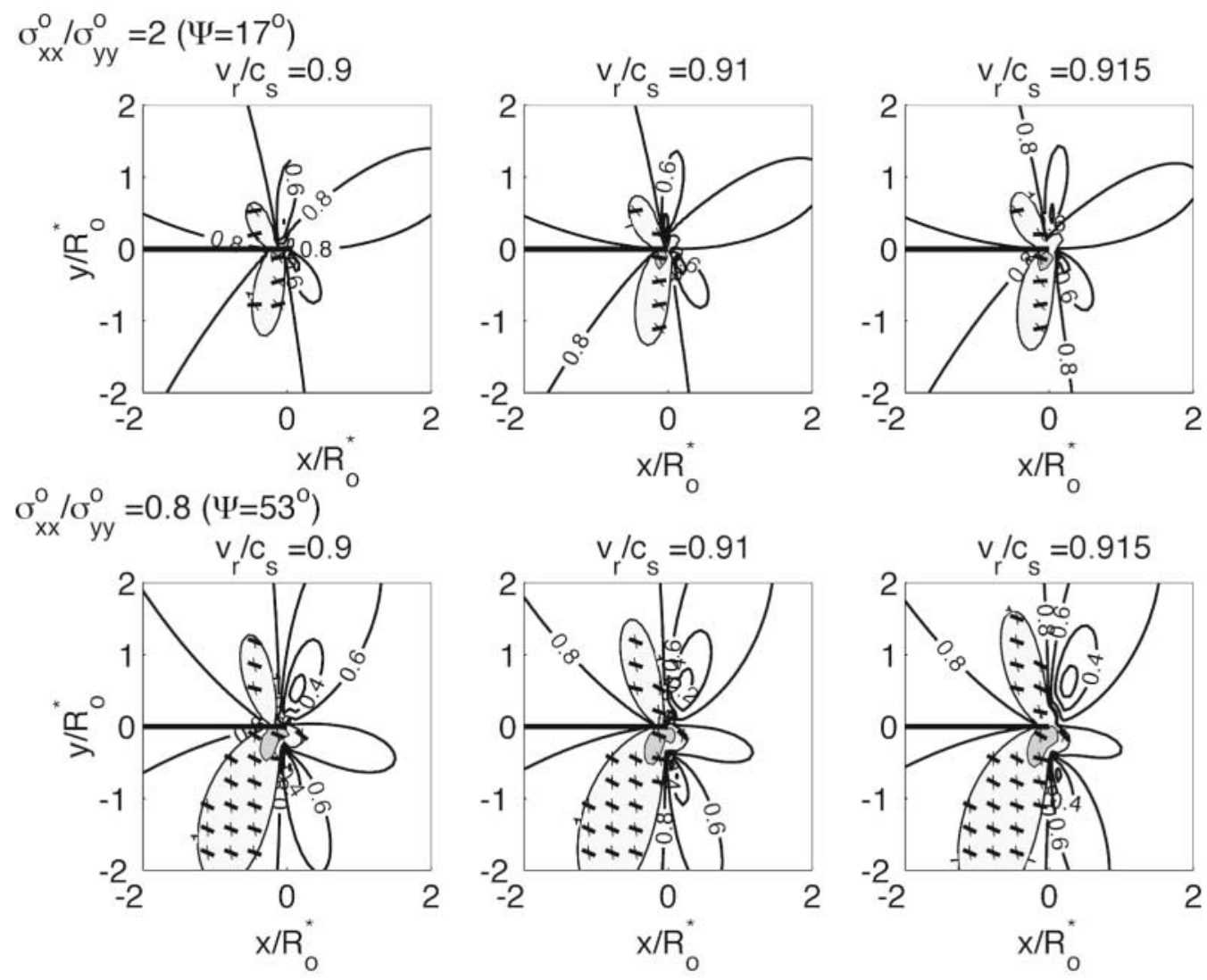

Figure 9. The same as Figure $8(R / L=0.9)$ except that the three scaled velocities are now $v_{r} / c_{s}=0.9,0.91$, and 0.915 . Note that the off-fault stress field only increases slightly as the limiting value of $v_{r} / c_{s} \rightarrow v_{r} / c_{R} \approx 0.92$.

weakening distance $\delta_{1}$, and $R_{o}^{*}$. The seismic moments and fault parameters for these seven events are also given in Table 1. Our estimates of $G, \sigma_{x y}^{o}-\tau_{r}, \delta_{1}$, and $R_{o}^{*}$ were calculated as described in the following sections and are summarized in Table 2.

\section{Fracture Energy}

The fracture energy was estimated for each event in Table 1 by using

$$
G=G^{*}\left(\frac{\mu \delta^{2}}{\pi L}\right)
$$

where $G^{*}$ is given by equation (20) and Figure 15. The values of $G_{\min }$ in Table 2 are minimum values in that they were calculated assuming $R / L=0$. They could be twice as large, $G_{\max }=2 G_{\min }$, at the other limit $R / L=1$ (see Fig. 15). We must accept that $R / L$ is an unknown quantity (which is to say that the ratio of stress drop to strength drop is unknown), so the best we can do within our model, for given values of $L, \delta$, and $v_{r}$, is to assert that the actual $G$ lies between $G_{\min }$ and $G_{\max }$. Values for $G_{\min }$ range from 0.1 to $4.6 \mathrm{MJ} / \mathrm{m}^{2}$ with an average of $1.9 \mathrm{MJ} / \mathrm{m}^{2}$. Thus, including the factor-of-two model uncertainty (we are not in a position to address uncertainty in the seismic inversion or limitations from our use of a 2D steady-state model), we conclude that $G$ falls in the range 0.1 to $9 \mathrm{MJ} / \mathrm{m}^{2}$ for the various events, with average between 2 and $4 \mathrm{MJ} / \mathrm{m}^{2}$. The larger values of $G$ tend to be for the larger events. The lowest $G_{\min }$ value of $0.1 \mathrm{MJ} / \mathrm{m}^{2}$ for the North Palm Springs earthquake is largely a consequence of its reported high rupture velocity (see Table 1), very near $c_{\text {lim }}$. Rice (2000) estimated a more narrow range, quoted also in PDR, because he assumed a uniform $v_{r}=$ $0.85 c_{s}$ for all events, thus giving for example, $G_{\min }=0.3$ $\mathrm{MJ} / \mathrm{m}^{2}$ for North Palm Springs.

\section{Dynamic Stress Drop}

Equation (A23) was used to calculate the dynamic stress drop as $\sigma_{y x}^{o}-\tau_{r}=G / \delta$. In fact, the dimensionless $G^{*}$ of (20) has the alternative interpretation $\sigma_{y x}^{o}-\tau_{r}=G^{*} \mu \delta / \pi L$. The dynamic stress drops based on $G_{\min }$ (for $R / L=0$ ) range from 0.3 to $3.3 \mathrm{MPa}$ with an average of $1.9 \mathrm{MPa}$. There was no evident trend with event size. Again, these are minimum values. They could be twice as large if we used $G_{\max }(R / L$ $=1$ ), and the best we can do is say that they lie between the numbers in the table and twice those numbers. 


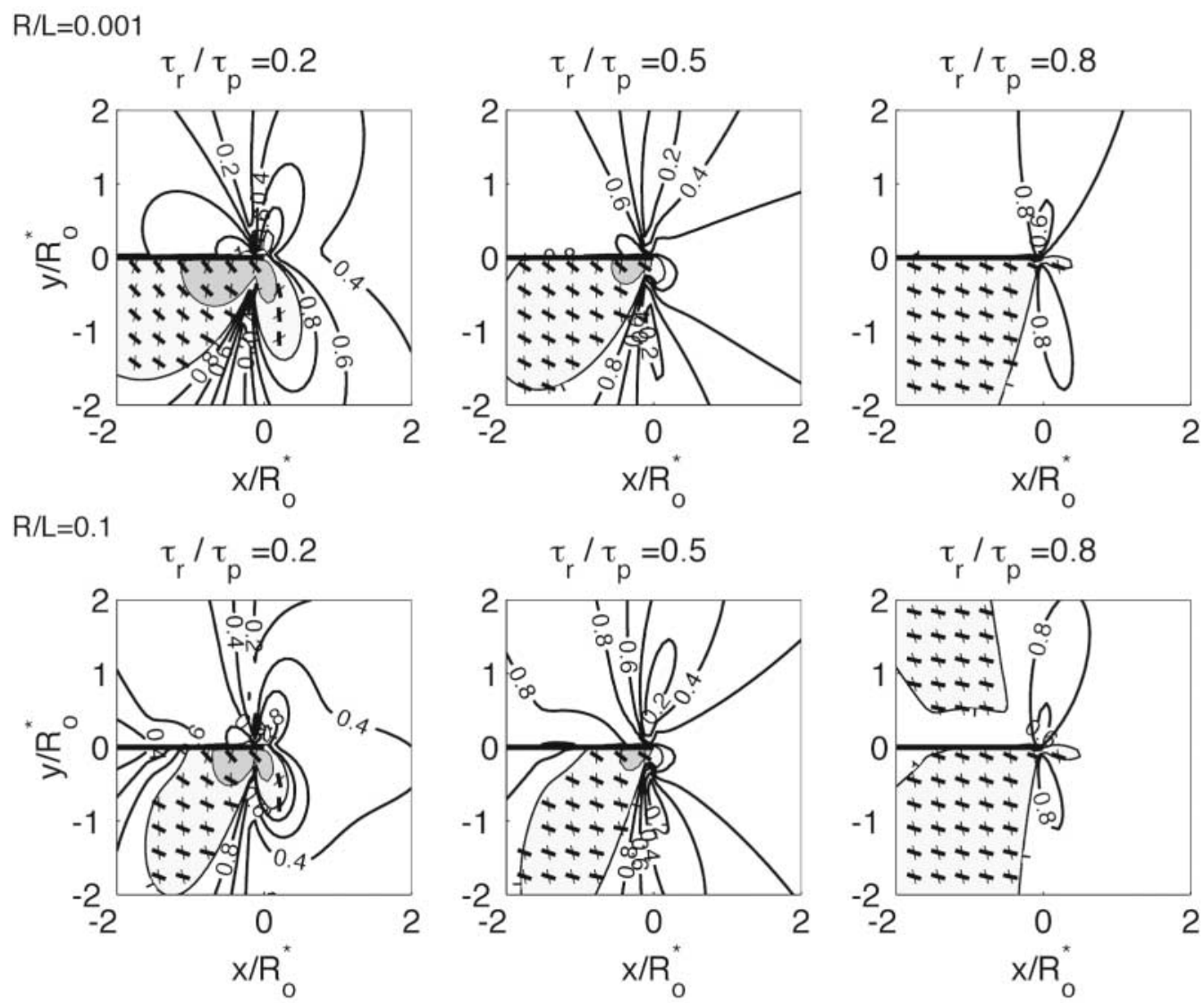

Figure 10. Contour plot of $\tau_{\max } / \tau_{\text {Coulomb }}$ as a function of position surrounding the tip of a rapidly propagating slip pulse $\left(v_{r} / c_{s}=0.9\right.$ in all cases) for a range of residual strength ratios $\tau_{r} / \tau_{p}=0.2,0.5$, and 0.8 and for $\sigma_{x x}^{o} / \sigma_{y y}^{o}=1$ (so that $\Psi=45^{\circ}$ ). The top row is for $R / L=0.001$ (scaled stress drop $=0.013$ ), which produces results essentially identical with figure 13 in Poliakov et al. (2002) for which $L \rightarrow \infty$ and $R / L$ $\rightarrow 0$. The bottom row is for $R / L=0.1$ (scaled stress drop $=0.136$ ). Again, $B=0$; Figure 12 to follow gives results corresponding to the bottom row here when $B=0.3$ and $B=0.6$

\section{Slip-Weakening Displacement}

The fracture energy $G$ can be interpreted as the area between $\tau_{p}$ and $\tau_{r}$ under the curve of shear stress versus displacement, as the shear stress drops from $\tau_{p}$ to $\tau_{r}$ with increasing displacement $\Delta u$. If we assume an exponential form for this decrease,

$$
\tau(\Delta u)=\left(\tau_{p}-\tau_{r}\right) \exp \left(-\Delta u / \delta_{1}\right)+\tau_{r}
$$

where $\delta_{1}$ is some constant characteristic slip for the weakening process, the integration yields $G=\left(\tau_{p}-\tau_{r}\right) \delta_{1}$. A weakening displacement $\delta_{1}$ defined by that relation to $G$ is seen, using (A23), to be in the same ratio to the locked in displacement $\delta$ as is the stress drop to the strength drop, that is, $\delta_{1} / \delta=\left(\sigma_{y x}^{o}-\tau_{r}\right) /\left(\tau_{p}-\tau_{r}\right)$. Note that this exponential form in (22) agrees with the Lachenbruch (1980) analysis of weakening by thermal pressurization of a pore fluid in the deforming fault gouge in the undrained, adiabatic limit (see also Sibson, 1973; Mase and Smith, 1987; Andrews, 2003). Then, $\tau_{r}$ is proportional to the ratio of dilation to shear rates in the gouge, assumed constant by Lachenbruch. Using thermal properties of water and granite at $100 \mathrm{MPa}$ pressure and $300^{\circ} \mathrm{C}, \delta_{1}$ is predicted (Rice, 2003) to be $1.7(1+r) / f$ times the thickness $h$ of the deforming gouge layer. Here, $f$ is the friction coefficient prevailing during large slip and $r$, neglected in the Lachenbruch analysis, is the ratio of fractional volume change of pore space per unit pore pressure increase divided by the compressibility of the pore fluid (called $\beta_{\phi} /$ $\beta_{f}$ in Segall and Rice [1995]); $r$ is likely to be of the order 1 to 2 . Thus, the adiabatic undrained weakening model predicts $\delta_{1} \approx 7 h$ if $f=0.6$, and $\delta_{1} \approx 20 h$ if $f=0.2$.

We first estimate $\left(\tau_{p}-\tau_{r}\right)$ for each earthquake by assuming $\tau_{p} \gg \tau_{r}$ (as implied by the absence of significant heat generation by large faults [Lacenbruch and Sass, 1980; Zoback et al., 1988]) and calculating $\tau_{p}=0.6 \bar{\sigma}$, where $\bar{\sigma}=$ [overburden - hydrostatic $p]_{\bar{z}}$ is the effective normal stress calculated at the median depth $\bar{z}$ of each earthquake rupture. Alternatively, it may be said that we simply assume $\tau_{p}-\tau_{r}=0.6 \bar{\sigma}$. The characteristic slip-weakening displacement, calculated as $\delta_{1} \approx G /(0.6 \bar{\sigma})$, then ranges from 1 to 49 


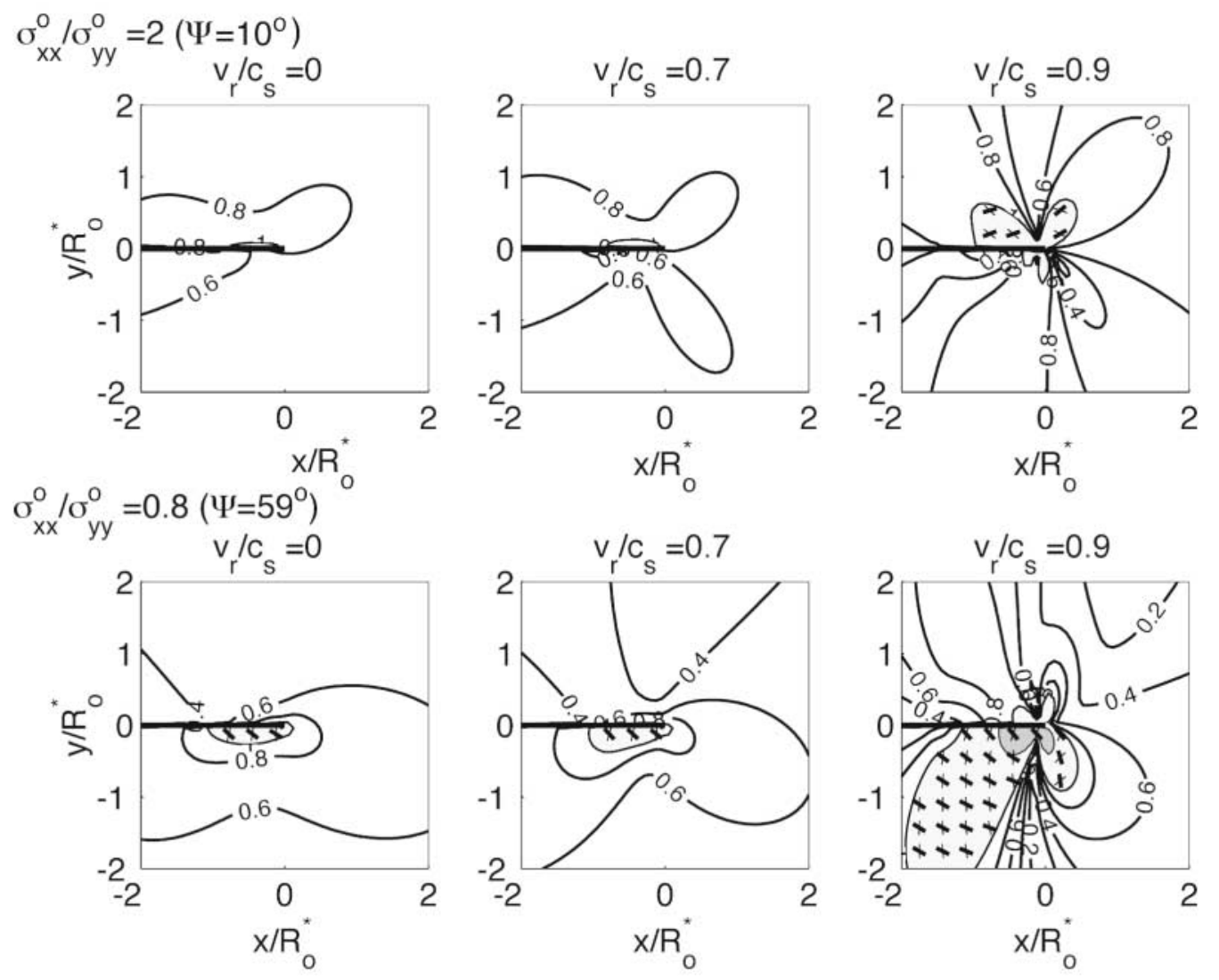

Figure 11. This is for the same set of parameters as in Figure 6 except that poroelastic coupling is included here, with $B=0.6$, whereas $B=0$ in Figure $6(R / L=$ 0.1 , scaled stress drop $=0.136 ; \tau_{r} / \tau_{p}=0.2$; the columns are for propagation velocities $v_{r} / c_{s}=0,0.7$, and 0.9 ; in the top row $\sigma_{x x}^{o} / \sigma_{y y}^{o}=2$ and $\Psi=10^{\circ}$, and in the bottom row $\sigma_{x x}^{o} / \sigma_{y y}^{o}=0.8$ and $\Psi=59^{\circ}$ ).

mm based on $G_{\min }$; again, because $G_{\min } \leq G \leq G_{\max }=$ $2 G_{\min }$, actual values could be up to a factor of 2 higher, and the total range is therefore 1 to $98 \mathrm{~mm}$. Within the undrained adiabatic interpretation with $f=0.6$ previously, these would imply a thickness $h$ of the deforming gouge zone on the order of 0.1 to $15 \mathrm{~mm}$ (the smaller end of that range could not be consistent with absence of fluid and heat transport). If we assumed a much lower friction during rapid slip, for example, due to flash heating (Rice, 1999; Tullis and Goldsby, 2002) of, say, $f=0.2$, so that $\delta_{1} \approx G /\left(0.2 \bar{\sigma}_{n}\right)$, the estimated range of $\delta_{1}$ triples over that previously, to 3 to $290 \mathrm{~mm}$, but the corresponding range of thickness $h$ does not change, because it also scales as $1 / f$.

We may also calculate $\delta_{1}$ for the case of a very low strength drop, where we just assume $\left(\tau_{p}-\tau_{r}\right)=10 \mathrm{MPa}$ for all events. Then $\delta_{1}$ ranges from 10 to $460 \mathrm{~mm}$ based on $G_{\text {min }}$. Again, there is the factor-of-two range for $G$ so that each $\delta_{1}$ could be up to 2-fold larger and the actual range for $\delta_{1}$ is 10 to $920 \mathrm{~mm}$.

We have treated $G$ here as being entirely due to slip weakening. However, our analysis of stress fields off the main fault plane suggests (Figs. 5-8, 11, 13, and 14) that as $v_{r}$ increases over the typical range inferred for rupture speeds, from 0.7 to $0.9 c_{s}$, inelastic deformation and secondary failures at locations off the main fault plane will begin, or will increase markedly in spatial extent. This process must also contribution to the fracture energy and would seem to add a component of $G$, which rises rather rapidly with increase of $v_{r}$ over that range. That might typically make fracture speeds self-limiting so that a propagation speed is chosen finitely below $c_{\text {lim }}$, as already suggested by PDR. The process requires a more precise analysis, in which full account is taken of the coupling (ignored here) between nonelastic deformation off the fault plane and redistribution of the stress field there.

\section{Slip-Weakening Zone Size}

All distances in the preceding calculations are scaled by the size $R_{o}^{*}$ of the displacement-weakening zone in the limit of low scaled stress drop and low rupture velocity. Because our calculations found that Coulomb failure extends to a distance on the order of $R_{o}^{*}$ (how far depends, especially, on $v_{r}$ but also on the prestress state and ratio of residual to peak strength; see Figs. 5-14), we estimate $R_{o}^{*}$ for each earthquake using equation (13). Two cases are considered: 
$B=0.3$
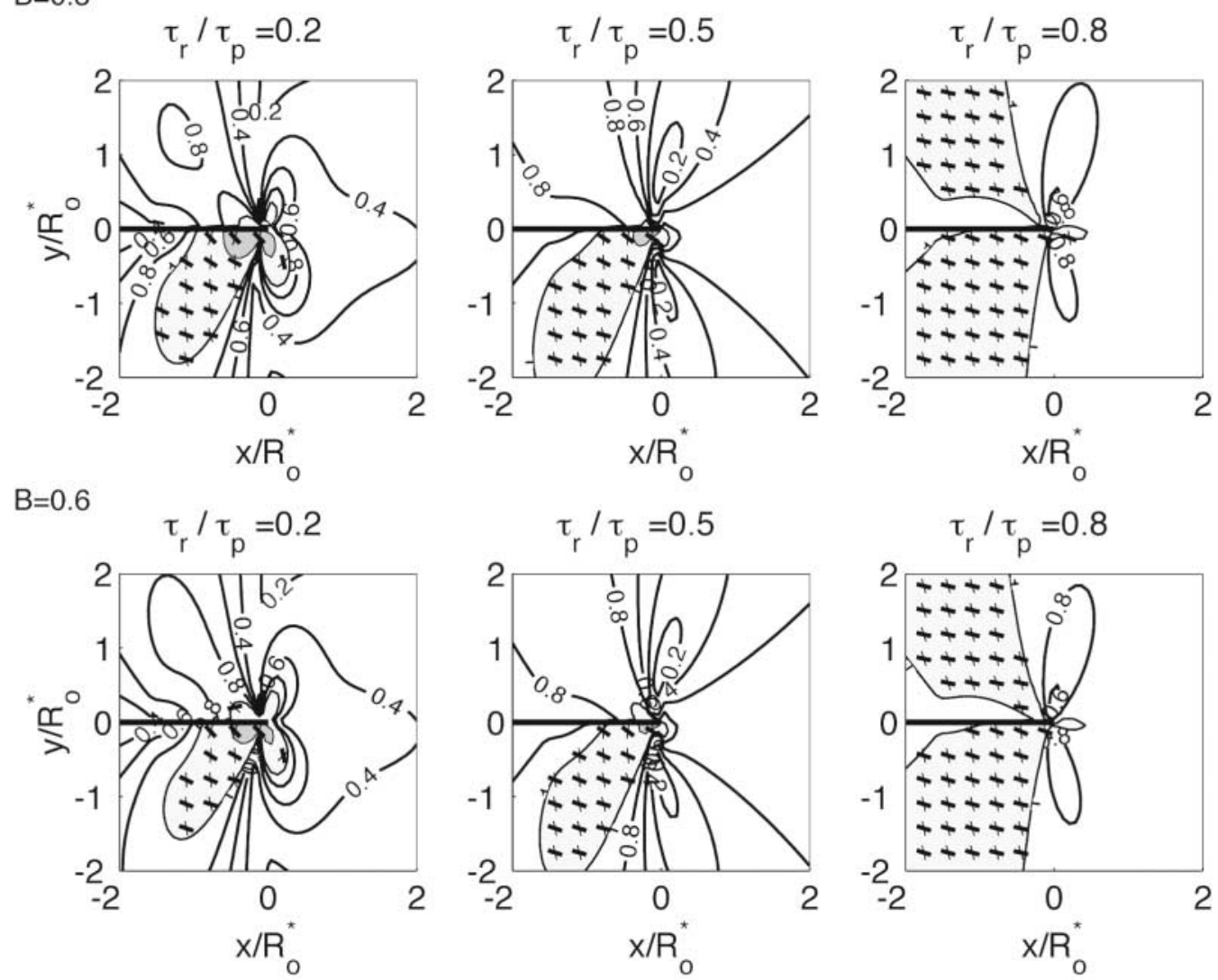

Figure 12. Porelastic coupling results with $B=0.3$ in the upper row and $B=0.6$ in the bottom row. This is for $v_{r} / c_{s}=0.9, R / L=0.1$ (scaled stress drop $=0.136$ ), and $\sigma_{x x}^{o} / \sigma_{y y}^{o}=1\left(\Psi=45^{\circ}\right)$, and for the three strength ratios $\tau_{r} / \tau_{p}=0.2,0.5$, and 0.8 . Those parameters other than $B$ are the same as in the bottom row of Figure 10, which thus provides the case $B=0$ for comparison.

(a) high peak strength and low dynamic strength $\left(\tau_{p} \gg \tau_{r}\right)$ such that $\left(\tau_{p}-\tau_{r}\right) \approx \tau_{p}=0.6 \bar{\sigma}_{n}$, and (b) low strength drop, $\tau_{p}-\tau_{r}=10 \mathrm{MPa}$ for all depths. For case (a) of high peak strength, listed in Table 2, $R_{o}^{*}$ ranged from 1.3 to $36 \mathrm{~m}$ based on $G_{\min }$, and for the same reasons previously stated each entry could be up to two-fold larger, so that the range is 1.3 to $72 \mathrm{~m}$. For the low strength case (b), $R_{o}^{*}$ ranged from $73 \mathrm{~m}$ to $3.3 \mathrm{~km}$ based on $G_{\min }$, and again each could be twice as large. These unusually large values for $R_{o}^{*}$ in case (b) reflect that $R_{o}^{*}$ is sensitive to the strength drop, varying as $\left(\tau_{p}\right.$ $\left.\tau_{r}\right)^{-2}$ in equation (13).

\section{The Effect of Depth}

One interesting question is how do the parameters in Table 2 vary with depth? It is commonly assumed that $\delta_{1}$ is approximately independent of depth because this parameter is associated with some physical length scale like the thickness of the active gouge zone (Lachenbruch, 1980). Because $G \approx 0.6 \bar{\sigma}_{n} \delta_{1}$, the fracture energy increases with depth as $\bar{\sigma}$. For the high-strength case, the strength drop also increases with depth as (see equation 30) $\tau_{p}-\tau_{r} \approx 0.6 \bar{\sigma}_{n}$. Hence, $R_{o}^{*}$ decreases with depth as

$$
R_{o}^{*}=\frac{9 \pi}{16(1-v)} \frac{\mu G}{\left(\tau_{p}-\tau_{r}\right)^{2}} \approx \frac{9 \pi}{16(1-v)} \frac{\mu \delta_{1}}{0.6 \bar{\sigma}_{n}}
$$

for mode II and we expect the size of the Coulomb failure zone, which is on the order of $R_{o}^{*}$ (see previously), to also decrease as $\bar{\sigma}_{n}^{-1}$. If the active thickness of the gouge also decreases with depth, then $R_{o}^{*}$ will be less sensitive to depth. Recall, the numbers reported in the table for $R_{o}^{*}$ refer to a median depth $\bar{z}$ along the rupture zone; it should be higher near the surface and lower at the base of the rupture zone.

\section{Discussion}

The rupture model developed here is a 2D dynamic slippulse model in which slip is limited to a finite zone of length $L$ behind a crack tip that moves at constant velocity $v_{r}$. Our model represents an improvement or at least an enlargement on previous slip-pulse models (Broberg, 1978; Freund, 1979 ) in that it includes a more explicit account of slipweakening friction and characterization of the off-fault stress state. We have extended the dynamic rupture model developed by PDR, which also includes slip-weakening, but which 

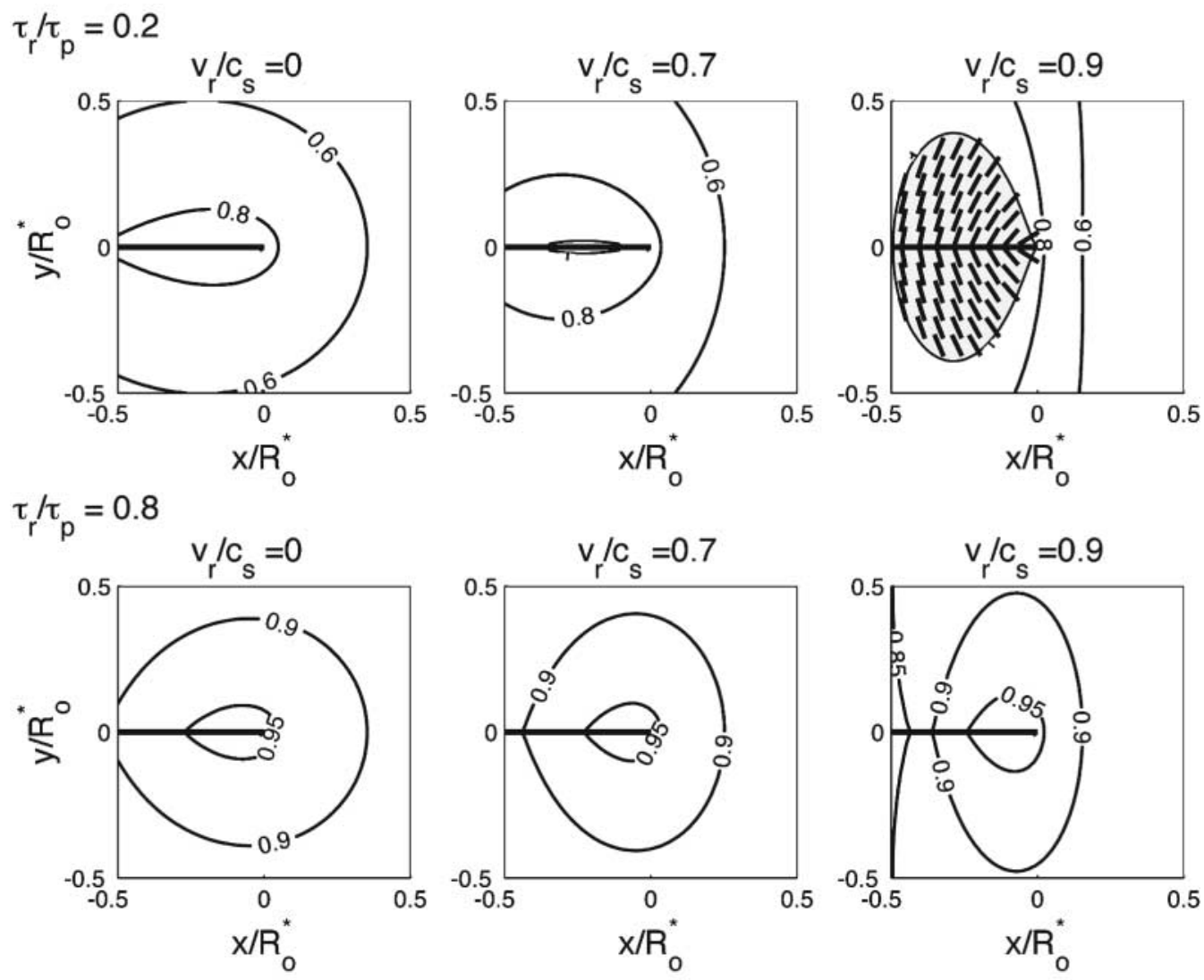

Figure 13. Contour plot of $\tau / \tau_{p}$, where $\tau=$ is the shear stress and $\tau_{p}$ is the static strength. Areas of off-fault slip $\left(\tau / \tau_{p}>1\right)$ are shaded and line segments indicate the orientations of planes of maximum shear. The three columns of plots are for three values of the scaled rupture velocity $v_{r} / c_{s}=0,0.7$, and 0.9 . The top row is for $\tau_{r} / \tau_{p}$ $=0.2$, the bottom row is for $\tau_{r} / \tau_{p}=0.8$. The very small value of $R / L=0.001$ (scaled stress drop $=0.013$ ) assumed here reproduces figure 15 in Poliakov et al. (2002), where $L \rightarrow \infty$.

models a semi-infinite slipping zone (i.e., $L / R \rightarrow \infty$ ) and dynamic stress drop that is very much smaller than the strength drop $\left(\left[\sigma_{y x}^{o}-\tau_{r}\right] /\left[\tau_{p}-\tau_{r}\right] \rightarrow 0\right)$. As such, the PDR model does not describe the extent of off-fault damage surrounding a propagating slip pulse over a wide range of parameters of interest. The discrepancy is particularly large as $v_{r}$ approaches its limiting velocity where the damage zone predicted by the PDR model grows very large, whereas that predicted by the slip-pulse model with finite, non-zero $\left(\sigma_{y x}^{o}-\tau_{r}\right) /\left(\tau_{p}-\tau_{r}\right)$ approaches a smaller asymptotic value.

\section{Static Stress Drop, Radiated Energy}

Our 2D steady-state model, strictly interpreted, cannot be used to estimate static stress drop on the fault plane or radiated seismic energy. It predicts zero for both. The static stress drop vanishes because the stress on the fault plane grows from $\tau_{r}$ back to $\sigma_{y x}^{o}$ behind the slip pulse (see Fig. 2). This is a direct consequence of the infinite extent of our fault plane in the $z$ direction. For energy, there is no change with time of total strain or kinetic energy, and all the work of remote stress in an increment of time is dissipated as the work against fault plane stresses resisting slip (equations A22 and A23), implying no radiated energy.

However, relative to an unsteady 3D description, our 2D model can be regarded as a local representation of a narrow slip-pulse region that separates the part of the fault that has not yet slipped from that which has slipped and is now relocked, for general, realistic shapes of that latter part. For example, the slipped and now relocked zone could grow as a circular region of expanding radius, or as some more general expanding oval or rectangular shape, just as in standard kinematic dislocation models of rupture (Aki and Richards, 1980). Dislocation rise time would then be identified as slip-pulse duration, that is, as $L / v_{r}$ in our notation, and a properly non-zero static stress drop and radiated energy could then be calculated by standard methods. The steady 2D model that we consider would then seem an appropriate representation if that rise time is short compared with a characteristic time, say $L /|\dot{L}|$, over which $L$ changes. That condition just requires $|\dot{L}| \ll v_{r}$, a condition that may often be met. 
$\tau_{r} / \tau_{p}=0.2$

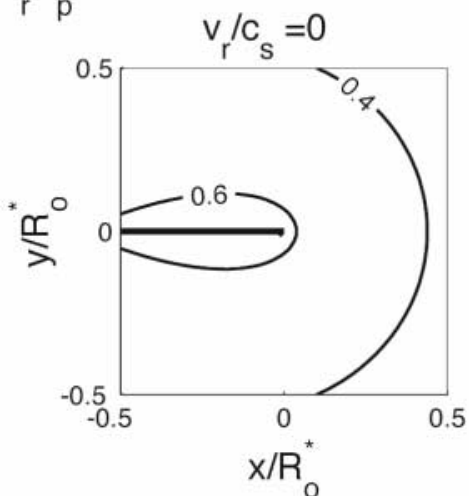

$\tau_{r} / \tau_{p}=0.8$

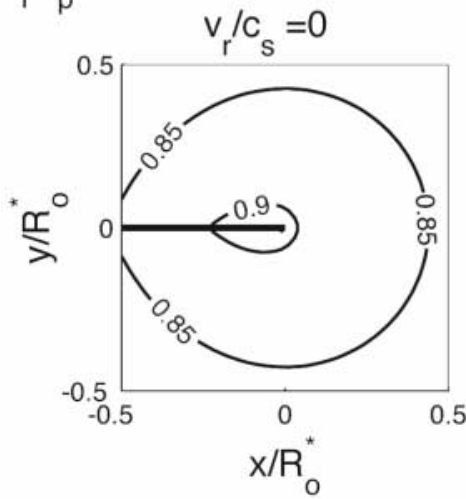

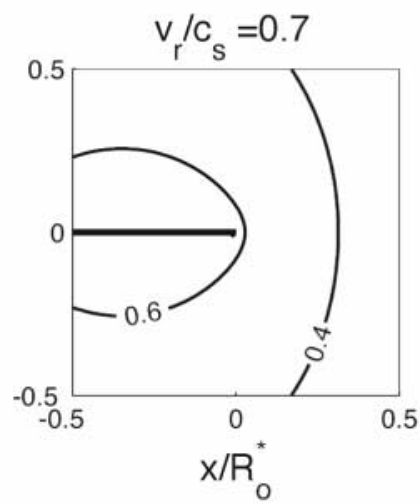
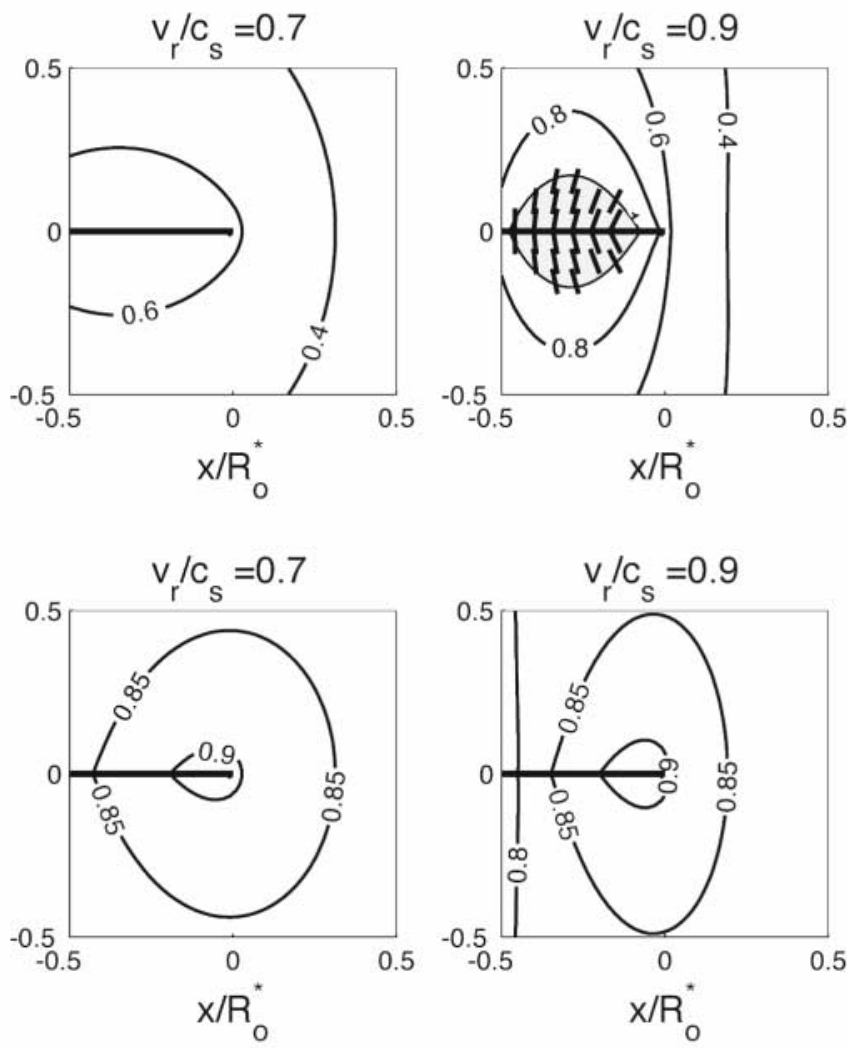

Figure 14. The same as Figure 13 except that $R / L=0.5$ (scaled stress drop $=$ $0.318)$. This larger value results in less extensive off-fault failure.

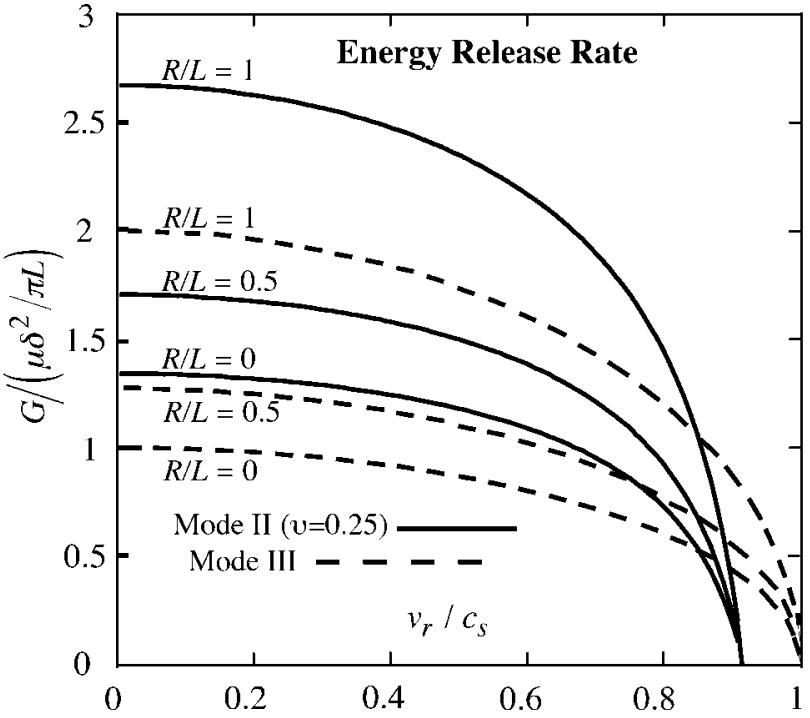

Figure 15. Scaled energy release rate as a function of rupture speed for a range of $R / L$. The $R / L$ values of $0,0.5$ and 1 correspond to scaled stress drops of $0,0.318$, and 0.5 , respectively.
Table 1

Earthquake Source Parameters from Heaton (1990)

\begin{tabular}{lccccccc}
\hline Event & $\begin{array}{c}M_{o} \\
\left(10^{18} \mathrm{~N}\right)\end{array}$ & $\begin{array}{c}l \\
(\mathrm{~km})\end{array}$ & $\begin{array}{c}w \\
(\mathrm{~km})\end{array}$ & $\begin{array}{c}\delta \\
(\mathrm{m})\end{array}$ & $\begin{array}{c}v_{r} \\
(\mathrm{~km} / \mathrm{s})\end{array}$ & $\begin{array}{c}T_{s} \\
(\mathrm{~s})\end{array}$ & $\begin{array}{c}L \\
(\mathrm{~km})\end{array}$ \\
\hline $\begin{array}{c}\text { Michoacan 1985 }(M \text { 8.1) } \\
\text { depth range, 10-35 km, est. }\end{array}$ & 1500 & 150 & 120 & 2.8 & 2.6 & 5 & 13 \\
$\begin{array}{c}\text { Borah Peak 1983 }(M 7.3) \\
\text { depth range, 0-15 km, est. }\end{array}$ & 23 & 40 & 20 & 0.96 & 2.9 & 0.6 & 1.7 \\
$\begin{array}{c}\text { San Fernando 1971 }(M \text { 6.5) } \\
\text { depth range, 3-15 km }\end{array}$ & 7 & 12 & 14 & 1.4 & 2.8 & 0.8 & 2.2 \\
$\begin{array}{c}\text { Imperial Valley 1979 }(M \text { 6.5) } \\
\text { depth range, 0-10 km }\end{array}$ & 5 & 30 & 10 & 0.56 & 2.6 & 1.0 & 2.6 \\
$\begin{array}{c}\text { Morgan Hill 1984 }(M \text { 6.2) } \\
\text { depth range, 0-12 km }\end{array}$ & 2.1 & 20 & 8 & 0.44 & 2.8 & 0.3 & 0.8 \\
$\begin{array}{c}\text { North Palm Springs 1986 }(M \text { 6.0) } \\
\text { depth range, 1-12 km }\end{array}$ & 1.8 & 18 & 10 & 0.33 & 3.0 & 0.4 & 1.2 \\
$\begin{array}{c}\text { Coyote Lake 1979 }(M \text { 5.9) } \\
\text { depth range, 3-10 km }\end{array}$ & 0.35 & 6 & 6 & 0.32 & 2.8 & 0.5 & 1.4 \\
\hline
\end{tabular}

$M_{o}$, seismic moment; $v_{r}$, rupture propagation speed; $l, w$, length, width of rupture area; $T_{s}$, duration of slip; $\delta$, slip left in wake of pulse; $L$, length of slipping zone, $L=v_{r} T_{s}$. est., estimated. 
Table 2

Fracture Parameters for the Slip-Pulse Model Calculated Using the Data from Heaton (1990) Summarized in Table 1

\begin{tabular}{lcccc}
\hline Event & $\begin{array}{c}G_{\min } \\
\left(\mathrm{MJ} / \mathrm{m}^{2}\right)\end{array}$ & $\begin{array}{c}\left(\sigma_{y x}^{o}-\tau_{r}\right)_{\min } \\
(\mathrm{MPa})\end{array}$ & $\begin{array}{c}\left(\delta_{1}\right)_{\min } \\
(\mathrm{mm})\end{array}$ & $\begin{array}{c}\left(R_{o}^{*}\right)_{\min } \\
(\mathrm{m})\end{array}$ \\
\hline $\begin{array}{c}\text { Michoacan 1985 }(M \text { 8.1) } \\
\text { depth range, 10-35 km, est. }\end{array}$ & 4.4 & 1.6 & 15 & 3.8 \\
$\begin{array}{c}\text { Borah Peak 1983 }(M 7.3) \\
\text { depth range, 0-15 km, est. }\end{array}$ & 1.9 & 2.0 & 23 & 19 \\
$\begin{array}{c}\text { San Fernando 1971 }(M \text { 6.5) } \\
\quad \text { depth range, 3-15 km }\end{array}$ & 4.6 & 3.3 & 49 & 36 \\
$\begin{array}{c}\text { Imperial Valley 1979 }(M \text { 6.5) } \\
\quad \text { depth range, 0-10 km }\end{array}$ & 0.88 & 1.6 & 17 & 22 \\
$\begin{array}{c}\text { Morgan Hill 1984 }(M \text { 6.2) } \\
\text { depth range, 0-12 km }\end{array}$ & 1.3 & 2.9 & 20 & 22 \\
$\begin{array}{c}\text { North Palm Springs 1986 }(M 6.0) \\
\text { depth range, 1-12 km }\end{array}$ & 0.10 & 0.31 & 1.4 & 1.3 \\
$\begin{array}{c}\text { Coyote Lake 1979 }(M \text { 5.9) } \\
\text { depth range, 3-10 km }\end{array}$ & 0.38 & 1.2 & 6.0 & 6.6 \\
\hline
\end{tabular}

Assumes $\mu=30 \mathrm{GPa}, \rho=2800 \mathrm{~kg} / \mathrm{m}^{3}, c_{s}=3.3 \mathrm{~km} / \mathrm{s}$. When $R / L=$ $0, G=G_{\min } \equiv G^{*} \mu \delta^{2} /(\pi L), \sigma_{y x}^{o}-\tau_{r}=\left(\sigma_{y x}^{o}-\tau_{r}\right)_{\min }=G_{\min } / \delta$. When $R / L=1, G=G_{\max }=2 G_{\min }, \sigma_{y x}^{o}-\tau_{r}=\left(\sigma_{y x}^{o}-\tau_{r}\right)_{\max }=2\left(\sigma_{y x}^{o}-\tau_{r}\right)_{\min }$. $\delta_{1}\left(=G /\left(\tau_{p}-\tau_{r}\right)\right)$ and $R_{o}^{*}\left(=(3 \pi / 4) \mu G /\left(\tau_{p}-\tau_{r}\right)^{2}\right)$ were calculated for the case $\tau_{p}-\tau_{r}=f_{s} \bar{\sigma}_{n}$ (i.e., assuming $\tau_{p} \gg \tau_{r}$ ) with $f_{s}=0.6$ and $\bar{\sigma}_{n}=$ overburden - hydrostatic pore pressure at middle of depth range; for other choices of $f_{s}$, note that $\delta_{1} \alpha 1 / f_{s}$ and $R_{o}^{*} \alpha 1 / f_{s}^{2}$. Values shown are for $G=$ $G_{\min }(R / L=0)$ and would double for $G=G_{\max }=2 G_{\min }(R / L=1)$. est., estimated.

\section{Fracture Energy Estimates and Comparisons}

Our estimates of $G$ are 0.1 to $9 \mathrm{MJ} / \mathrm{m}^{2}$, with an average of $2-4 \mathrm{MJ} / \mathrm{m}^{2}$, for the large events characterized by Heaton (1990) from seismic slip inversions. Those estimates fall at the high end of the very broad range $\left(1 \mathrm{~J} / \mathrm{m}^{2}\right.$ to $\left.1 \mathrm{MJ} / \mathrm{m}^{2}\right)$ inferred by Husseini et al. (1975) in the first study of seismic estimates of $G$. We may make the following comparisons with more recent studies. For the 1984 Morgan Hill rupture, Beroza and Spudich (1988) used results of their slip inversion to infer an average $G \approx 2 \mathrm{MJ} / \mathrm{m}^{2}$, which is bracketed by our $G_{\min }$ to $G_{\max }$ range of $1.3-2.6 \mathrm{MJ} / \mathrm{m}^{2}$ for that event; for the 1979 Imperial Valley event, Favreau and Archuleta (2003) infer an average $G$ of $0.81 \mathrm{MJ} / \mathrm{m}^{2}$, whereas we find $0.88-1.9 \mathrm{MJ} / \mathrm{m}^{2}$. Our results, in general, are compatible with estimates of $G$ for other large events, not in the Heaton compilation. For example, from seismic inversions, Guatteri et al. (2001) estimate $1.5 \mathrm{MJ} / \mathrm{m}^{2}$ for the 1995 Kobe earthquake, consistent also with Ide (2002); Olsen et al. (1997) and Peyrat et al. (2001) estimate $5 \mathrm{MJ} / \mathrm{m}^{2}$ for the 1992 Landers earthquake. The results are also generally consistent with $\mathrm{MJ} / \mathrm{m}^{2}$ range estimates of $G$ for large events from analyses of large earthquake initiation and arrest (Aki, 1979; Li, 1987; Rudnicki and $\mathrm{Wu}, 1995)$.

Further, Abercrombie and Rice $(2001,2005)$ show that a quantity they call $G^{\prime}$ can be evaluated from the seismic stress drop (inferred from seismic moment and corner frequency or pulse duration) and radiated energy. Their $G^{\prime}$ coincides with $G$ when the final static shear stress on the fault is not very different from the dynamic friction stress during the last increments of slip, that is, the case of negligible dynamic overshoot or undershoot. They present arguments as to why such effects are modest and suggest that $G$ might range from about $0.6 G^{\prime}$ to an unconstrained, but probably not large, amount greater than $G^{\prime}$. For events with slips between about 0.2 and $4 \mathrm{~m}$, roughly comparable with slips $\delta$ in the Heaton data set used here, they find a range of $G^{\prime}=$ 0.3 to $20 \mathrm{MJ} / \mathrm{m}^{2}$. Further, they find that $G^{\prime}$ increases with slip, at least for slips less than about $0.5 \mathrm{~m}$. (The data is insufficient to show if there is saturation at larger slips.) We find a similar trend. For example, including the $G_{\min }$ to $G_{\max }$ range, our $G$ values for the three smallest-slip events in the table $(0.32-0.44 \mathrm{~m})$ are $G=0.1-3 \mathrm{MJ} / \mathrm{m}^{2}$, whereas for the three largest slip events (of 0.96 to $2.8 \mathrm{~m}$ ) $G=2-9 \mathrm{MJ} / \mathrm{m}^{2}$. Abercrombie and Rice (2005) show that a consistent explanation of the scaling of $G$ with event size is provided if the slip-weakening relation is such that strength continues to decrease with slip $\Delta u$, but at an ever decreasing rate of weakening, $-d \tau / d(\Delta u)$. For example, they propose that their scaling can be described approximately by a slip-weakening relation in the form $\tau \approx C-24(\Delta u)^{0.28}$, at least for $\Delta u$ above about $0.5 \mathrm{~mm}$; here, stress is in megapascals, $\Delta u$ in meters, and $C$ is unconstrained by their analysis. Their associated expression for $G^{\prime}$ (in $\mathrm{MJ} / \mathrm{m}^{2}$ ) is $G^{\prime}=5.25(\Delta u)^{1.28}$, where $\Delta u$ is again in meters.

To compare with that expression for $G^{\prime}$, the three largest slip events of Heaton's set have an average slip of $1.72 \mathrm{~m}$, and the three smallest have an average slip of $0.36 \mathrm{~m}$. The power law expression for $G^{\prime}$ (for which our $1.72 \mathrm{~m}$ extends beyond their range of well-documented scaling) would predict that $G_{1.72 \mathrm{~m}}^{\prime} / G_{0.36 \mathrm{~m}}^{\prime} \approx 7.4$. We can find from Table 2 that our average $G_{\min }=3.6 \mathrm{MJ} / \mathrm{m}^{2}$ for the larger events and $0.59 \mathrm{MJ} / \mathrm{m}^{2}$ for the smaller, which shows a ratio of 6.1 (our $G_{\max }$ values have the same ratio). That is reasonably consistent with the scaling of Abercrombie and Rice (2005). The actual $G^{\prime}$ predicted by that scaling is somewhat close to our average $G_{\max }$ for each event set; that is, an average $G_{\max }$ of 7.2 and $1.2 \mathrm{MJ} / \mathrm{m}^{2}$ for the respective event sets with average slips of 1.72 and $0.36 \mathrm{~m}$. In comparison, their expression for $G^{\prime}$ gives 10.5 and $1.4 \mathrm{MJ} / \mathrm{m}^{2}$ for the respective slips of 1.72 and $0.36 \mathrm{~m}$. Thus our results, although for a different set of events, are roughly consistent with the scaling and, if we use $G_{\max }$, with the magnitude of what Abercrombie and Rice (2005) infer for $G^{\prime}$.

\section{Factors in Estimating Fracture Energy}

As noted, the fracture energies that we have estimated using this solution have good consistency with other seismic results and are consistent with the use of generally plausible parameters for shear zone thickness in the Lachenbruch (1980) and Mase and Smith (1987) analyses of thermal weakening by pressurization of pore water. (Other weakening processes, not seen in the slow, small slips of traditional laboratory studies, may be active too; Sibson, 1975; Spray, 1993, 1995; Chambon et al., 2002; Goldsby and Tul- 
lis, 2002.) However, a number of things could go wrong in making such seismic estimates of $G$ as we do:

1. There are uncertainties in the seismic inversions themselves. For example, seismic records, in general, are filtered in inversion studies at periods shorter than on the order of $1 \mathrm{~s}$, to remove effects of scattering; $1 \mathrm{~s}$ is the same order as the slip durations for most events listed in Table 1 .

2. A correspondence of the mechanism leading to the apparently self-healing slip inferred in natural examples (Heaton, 1990) to the mechanism introduced in our model cannot be confirmed. From the perspective of theoretical models, self-healing is not a direct outcome of rupture models like we consider with a slip-rate-independent friction stress (Zheng and Rice, 1998); in general, it must be imposed in such models by propagating arrest phases or other interactions with heterogeneities (Day, 1982; Johnson, 1990; Perrin et al., 1995; Beroza and Mikumo, 1996; Nielsen and Carlson, 2000; Nielsen and Madariaga, 2003). Self-healing can also occur when spatially nonuniform slip can alter normal stress across a fault plane, as for sliding between elastically dissimilar solids (Weertman, 1980; Andrews and Ben-Zion, 1997; Harris and Day, 1997; Cochard and Rice, 2000) or between certain foam rubbers (Brune et al., 1993). Such alteration of normal stress is, in principle, a generic consequence of nonlinear constitutive response off the fault plane (Cochard and Rice, 2000), although such a route to self-healing pulses has not yet been demonstrated. Finally, slip-rate dependence of friction (Cochard and Madariaga, 1994, 1996; Perrin et al., 1995; Beeler and Tullis, 1996; Nielsen and Carlson, 2000; Nielsen and Madariaga, 2003), at least if present under sufficiently low driving stress (Zheng and Rice, 1998), is a robust route to self-healing. Thus, the array of possible models for self-healing is great and we do not have sufficiently general solutions, analogous to that developed here, for those other mechanisms to compare with the observations.

3 . The constraint from slip inversions on rupture velocity $v_{r}$ is actually a constraint on the average velocity. We cannot rule out the possibility that there are very strong fluctuations in $v_{r}$ at periods shorter than those that can be resolved in the inversion. In such cases we may argue that the net energy flow $G$ to the crack tip is sensibly estimated by our procedure, but only some of that (say, $G_{\text {mat }}$ ) is actually dissipated in frictional processes near the rupture front and the rest (call it $G_{\text {rad }}$, so that $G=G_{\text {rad }}+G_{\text {mat }}$ ) is radiated out again from the crack tip as high-frequency stress waves. A simplified analysis of this effect (Rice, 2000) may be carried out in the context of a mode III singular crack $(R / L \rightarrow$ 0 , scaled stress drop $\rightarrow 0$ ). Let $v_{r}^{\prime}$ be the time-variable instantaneous rupture speed, which averages to $v_{r}$, and suppose, to make a simple tractable case, that $v_{r}^{\prime}$ fluctuates rapidly between two constant values

$$
v_{r}^{\prime}=\left\{\begin{array}{l}
\hat{v}_{r}, \text { very near } c_{s}, \text { for a fraction of the time } \\
0 \text { for the rest of the time. }
\end{array}\right.
$$

This might roughly represent fracturing through a highly segmented fault system in which the material energy adsorption $G_{\text {mat }}$ is low on individual segments, so that $\hat{v}_{r}$ gets quite near $c_{s}$, but rupture arrests at segment ends until stress waves radiated out from there nucleate new ruptures on neighboring segments (e.g., Harris and Day, 1993).

During such rapidly fluctuating motion of the crack tip that there is no energy flow per unit time to the crack tip when $v_{r}^{\prime}=0$, but there is an energy flow to inelastic processes at the crack tip per unit time during the rapid motion at $v_{r}^{\prime}=\hat{v}_{r}$, amounting to

$$
G_{\mathrm{mat}}=\sqrt{\frac{1-\hat{v}_{r} / c_{s}}{1+\hat{v}_{r} / c_{s}}} G_{\mathrm{rest}}
$$

per unit fractured area (Eshelby, 1969a,b; Freund, 1990). Here, $G_{\text {rest }}$ is the "rest" value of the energy release rate and is unaffected by the rapid fluctuations (Rice et al., 1994; Morrissey and Rice, 1998). The $G$ that we infer based on the average speed $v_{r}$ must bear the same relation to $G_{\text {rest }}$, namely,

$$
G=\sqrt{\frac{1-v_{r} / c_{s}}{1+v_{r} / c_{s}}} G_{\text {rest }} .
$$

By taking ratios and eliminating $G_{\text {rest }}$, we thus find that the fraction of energy flow actually absorbed by inelastic processes at the tip is

$$
\frac{G_{\mathrm{mat}}}{G}=\sqrt{\frac{1-\hat{v}_{r} / c_{s}}{1+\hat{v}_{r} / c_{s}}} \sqrt{\frac{1+v_{r} / c_{s}}{1-v_{r} / c_{s}}}
$$

and the remaining fraction $\left(G_{\mathrm{rad}} / G=1-G_{\mathrm{mat}} / G\right)$ is radiated out in the high-frequency wave field created by the rapid velocity fluctuations. Thus, although we may with some confidence estimate $G$, that puts only an upper bound on how much energy is absorbed by the material near the tip, and in this simple illustration $G_{\text {mat }}$ may be made an arbitrarily small fraction of $G$ by making $\hat{v}_{r}$ arbitrarily close to $c_{s}$. For example, if $v_{r}=0.80 c_{s}$ and $\hat{v}_{r}=0.99 c_{s}$, then $G_{\text {mat }} \approx 0.21 G$ and $G_{\text {rad }} \approx 0.79 G$.

\section{Damage Zones and Fault Gouge}

Although we used the PDR model primarily to explore fault branching, we can address the additional goal here of exploring the mechanics responsible for the formation of fragmented fault rock. The wall rocks of most faults are separated by one or more layers of fragmented rock called gouge or fault breccia. (See Ben-Zion and Sammis [2003] for a review of fault-zone structure.) Although there is considerable variation in the width of these layers and the extent of fragmentation within them, most faults with significant slip tend to have a structure described by Chester and Logan 
(1986, 1987), Chester et al. (1993), Evans and Chester (1995), Chester and Chester (1998), Schulz and Evans (2000), Lockner et al. (2000), Wibberley (2002), Wibberley and Shimamoto (2003), and Otsuki et al. (2003). A "core" of extremely finely fragmented rock (possibly altered to clay) is bordered by layers of more coarsely fragmented fault breccia. The core contains one or more planar surfaces which appear to have accommodated most of the slip, and which Chester and Chester (1998) refer to as "prominent fracture surfaces." Somewhat surprisingly, the layers of fault breccia that border the core appear to have accommodated little if any strain, despite the fact that brecciation often extends to the micron scale. Low strain is inferred from observations that relict structures from the host rock do not appear to be significantly sheared.

Most studies of fault gouge and breccia to date have focused on the particle-size distribution. Sammis et al. (1987) found that breccia from the Lopez Canyon Fault (a branch of the now extinct San Gabriel Fault in southern California) had a power law distribution of particle sizes consistent with a fractal structure. This was not the expected result, because existing fragmentation laws predicted either a lognormal or exponential Rosin-Ramler distribution (see, e.g., Prasher, 1987). To explain these observations, Sammis et al. (1987) proposed a new fragmentation model, which they dubbed "constrained comminution." In constrained comminution, particles are not free to move as they are in normal milling operations. Rather, they are fixed in position relative to other particles by the dense packing and high pressures in the subterranean faulting environment. Consequently, the probability of a particle fracturing is not dominated by its fragility as in other fragmentation models, but by its loading geometry from adjacent particles. Particles loaded by samesized neighbors have the highest fracture probability. The process starts eliminating the largest (and hence weakest) particles until all large same-sized neighbors are eliminated, leaving isolated large particles cushioned by surrounding smaller ones. The process then works its way down, eliminating smaller and smaller same-sized neighbors. The result is a granular mass in which no two neighbors are the same size at any scale. A fractal distribution having fractal dimension $D=1.6$ has this property; in fact, this is the fractal dimension found for the Lopez Canyon breccia. Constrained comminution has been observed in the double-shear friction apparatus of J. H. Dieterich at U.S. Geological Survey (Biegel et al., 1989) and simulated in computer studies (Steacy and Sammis, 1991).

The development of a fractal particle distribution has been shown to have important mechanical consequences. Biegel et al. (1989) found that the emergence of a fractal distribution is closely associated with the observed transition from velocity-strengthening friction (and stable sliding) to velocity-weakening friction (and possible stick-slip instabilities). Sammis and Steacy (1994) proposed the explanation that deformation in nonfractal distributions is accommodated mostly by the failure of particles which, as in triaxial laboratory experiments, is velocity strengthening. As a fractal distribution is developed it becomes increasingly difficult to fracture particles and, at the same time, reduced dilatation signals that it is easier to accommodate deformation by slip between particles. Because the resistance to slip increases with the age of the particle contacts, velocity weakening becomes possible and the macroscopic friction transitions from velocity strengthening associated with the fracture of particles to weakening associated with slip between them.

The reduced dilatation associated with the evolving fractal distribution also leads to shear localization on surfaces within the granular mass. From this perspective, the reason fault breccia has low strain is that it represents only the initial phase of fault formation. Breccia forms early in a fault's history as it grows by linking offset strands (Segall and Pollard, 1983) and removing geometric asperities (Power et al., 1988). If these asperities have a fractal size distribution (i.e., the fault surface has fractal roughness), Power et al. (1988) show that the scale of the geometrical mismatch grows with fault displacement in such a way that the thickness of the resultant gouge and breccia zone increases approximately linearly with displacement, as often observed (Scholz, 1987; Hull, 1988). However, only a relatively small strain on the order of about 3 is required to produce a fractal gouge (Biegel et al., 1989), after which strain localizes onto a prominent slip surface. Once strain has localized, the gouge layer is a relict structure playing no significant role in the accommodation of additional strain.

This view of the formation and mechanical significance of gouge has recently been challenged by Brune (2001), who proposed that fault breccia is formed by dynamic stress changes during an earthquake. In particular, he argues that gouge forms during the dynamic reduction of normal stress across a fault accompanying the propagation of a "wrinkle pulse," or dynamic pore pressure reduction, or any other opening type motion of the class recently proposed to allow faulting without the attendant generation of heat-thus solving the "heat-flow paradox" for the San Andreas fault. As evidence, Brune cites the apparent lack of significant strain, observations of "exploded grains" that have obviously failed in tension, and the orientation of small slip surfaces within the breccia that are aligned at high angles to the prominent slip surface. As mentioned previously, the apparent lack of strain is not unique to dynamic rupture. Nor is the observation of grains that have failed in tension since such grains were observed in double-shear friction experiments (Biegel et al., 1989) where all macroscopic stresses were compressive. Tensile failure in that case resulted from the failure of individual grains under bipolar loading by same-sized neighbors in the load-bearing ligands (stress chains) that transmit force in granular media. It should be noted that Brune's hypothesis is consistent with Sibson's (1986) observation that "explosion breccia" tends to form in dilatational fault jogs. However, this is an expected outcome in quasistatic deformation and does not require a dynamic opening-type mode of rupture. 
Our results show that the dynamic stress field from a propagating slip pulse can produce Coulomb slip on preexisting fractures which extends out to a scaled distance on the order of $1-2 R_{o}^{*}$ in the range of typical seismic rupture velocities, $v_{r}=0.7-0.9 c_{s}$. Using our model to analyze the slip pulses observed by Heaton (1990) for seven large events, we found that $R_{o}^{*}$ is in the range of 1 to $40 \mathrm{~m}$ if $\tau_{p}-\tau_{r}=$ $0.6 \bar{\sigma}_{n}$, and can be on the order of kilometers if the strength drop is low, for example, $\left(\tau_{p}-\tau_{r}\right) \approx 10 M P a$. These results are roughly consistent with observations by Wilson et al. (1999) that a zone of oriented microfracture damage extends to distances of about $30 \mathrm{~m}$ from the exhumed Punchbowl Fault in southern California, and that random microfractures are above the background level to distances of about $100 \mathrm{~m}$.

Coulomb slip on preexisting fractures is a necessary, but not sufficient, condition for compressive failure and fragmentation of crystalline rock. A damage mechanics analysis (such as Ashby and Sammis, 1990) is required to map the boundaries of fragmentation. However, under certain conditions, we found that the minimum principal stress $\sigma_{2}$ becomes positive (tensile), which would almost certainly produce failure and fragmentation. As is evident in Figure 5, tensile stress is favored by a preexisting maximum compression direction at a high angle $\psi$ to the fault plane, and a high value of the rupture velocity $v_{r}$. The extent to which the fault zone becomes narrower with depth depends on the depth dependence of $\delta_{1} /\left(\tau_{p}-\tau_{r}\right)$. If $\left(\tau_{p}-\tau_{r}\right)$ depends on the effective normal stress through a coefficient of friction, then the zone width should decrease linearly with depth.

\section{Acknowledgments}

This study was supported by NSF-EAR Awards 0105344 and 0125709 to Harvard, by NSF-EAR Award 9902901 to USC, and by the NSF/USGS Southern California Earthquake Center (SCEC). SCEC is supported through USGS cooperative agreement 02HQAG0008 and NSF cooperative agreement EAR-0106924. This is SCEC Contribution Number 732. We are grateful to J. W. Rudnicki for helping us improve the presentation, and for reviews by T. H. Heaton and R. Madariaga.

\section{References}

Abercrombie, R. E., and J. R. Rice (2001). Small earthquake scaling revisited: can it constrain slip weakening? (abstract), EOS Trans. Am. Geophys. Union 82, no. 47 (Fall Meeting Suppl.), S21E-04, F843.

Abercrombie, R. E., and J. R. Rice (2005). Can observations of earthquake scaling constrain slip weakening? Geophys. J. Int. (in press).

Aki, K. (1979). Characterization of barriers on an earthquake fault, J. Geophys. Res. 84, 6140-6148.

Aki, K., and P. G. Richards (1980). Quantitative Seismology: Theory and Methods, Freeman, New York.

Andrews, D. J. (1976a). Rupture propagation with finite stress in antiplane strain, J. Geophys. Res. 81, 3575-3582.

Andrews, D. J. (1976b). Rupture velocity of plane strain shear cracks, $J$. Geophys. Res. 81, 5679-5687.

Andrews, D. J. (2003). A fault constitutive relation accounting for thermal pressurization of pore fluid, J. Geophys. Res. 107, (no. B12), 2363, doi 10.1029/2002JB001942.

Andrews, D. J., and Y. Ben-Zion (1997). Wrinkle-like slip pulse on a fault between different materials, J. Geophys. Res. 102, 553-571.
Ashby, M. F., and C. G. Sammis (1990). The damage mechanics of brittle solids in compression, Pure Appl. Geophys. 133, 489-521.

Beeler, N. M., and T. E. Tullis (1996). Self-healing slip pulse in dynamic rupture models due to velocity-dependent strength, Bull. Seism Soc. Am. 86, 1130-1148.

Ben-Zion, Y., and C. G. Sammis (2003). Characterization of fault zones, Pure Appl. Geophys. 160, 677-715.

Beroza, G. C., and T. Mikumo (1996). Short slip duration in dynamic rupture in the presence of heterogeneous fault properties, J. Geophys. Res. 101, 22,449-22,460.

Beroza, G. C., and P. Spudich (1988). Linearized inversion for fault rupture behavior: application to the 1984 Morgan Hill, California, earthquake, J. Geophys. Res. 93, 6275-6296.

Biegel, R. L., C. G. Sammis, and J. H. Dieterich (1989). The frictional properties of a simulated gouge having a fractal particle distribution, J. Structural Geol. 11, 827-846.

Broberg, K. B. (1978). On transient sliding motion, Geophys. J. R. Astr. Soc. 52, 397-432.

Broberg, K. B. (1999). Cracks and Fracture, Academic Press, San Diego.

Brune, J. N. (2001). Fault-normal dynamic unloading and loading: an explanation for "non-gouge" rock powder and lack of fault-parallel shear bands along the San Andreas fault (abstract), EOS Trans. Am. Geophys. Union 82, no. 47 (Fall Meeting Suppl.), F854.

Brune, J. N., S. Brown, and P. A. Johnson (1993). Rupture mechanism and interface separation in foam rubber models of earthquakes: a possible solution to the heat flow paradox and the paradox of large overthrusts, Tectonophysics 218, 59-67.

Chambon, G., J. Schmittbuhl, and A Corfdir (2002). Laboratory gouge friction: seismic-like slip weakening and secondary rate- and stateeffects, Geophys. Res. Lett. 29, no. 10, doi 10.1029/2001GL014467.

Chester, F. M., and J. M. Logan (1986). Implications for mechanical properties of brittle faults from observations of the Punchbowl fault zone, California, Pure Appl. Geophys. 124, 79-106.

Chester, F. M., and J. M. Logan (1987). Composite planar fabric of gouge from the Punchbowl fault, California, J. Struct. Geol. 9, 621-634.

Chester, F. M., and J. S. Chester (1998). Ultracataclasite structure and friction processes of the Punchbowl fault, San Andreas system, California, Tectonophysics 295, 199-221.

Chester, F. M., J. P. Evans, and R. L. Biegel (1993). Internal structure and weakening mechanisms of the San Andreas fault, J. Geophys. Res. 98, 771-786.

Cochard, A., and R. Madariaga (1994). Dynamic faulting under rate-dependent friction, Pure Appl. Geophys. 142, 419-445.

Cochard, A., and R. Madariaga (1996). Complexity of seismicity due to highly rate dependent friction, J. Geophys. Res. 101, no. B11, 25,32125,336 .

Cochard, A., and J. R. Rice (2000). Fault rupture between dissimilar materials: Ill-posedness, regularization, and slip-pulse response, J. Geophys. Res. 105, no. B11, 25,891-25,907.

Day, S. M. (1982). Three-dimensional finite difference simulation of fault dynamics: rectangular faults with fixed rupture velocity, Bull. Seism. Soc. Am. 72, 705-727.

Erdogan, F. (1968). Crack-propagation theories, in Fracture: An Advanced Treatise, H. Liebowitz (Editor), Vol. 2. Mathematical Fundamentals, Academic Press, New York, 497-590.

Eshelby, J. D. (1969a). The elastic field of a crack extending non-uniformly under general anti-plane loading, J. Mech. Phys. Solids 17, 177.

Eshelby, J. D. (1969b). The starting of a crack, in Physics of Strength and Plasticity, A. S. Argon (Editor), The MIT Press, Cambridge, Massachusetts, 263-275.

Evans, J. P., and F. M. Chester (1995). Fluid-rock interaction in faults of the San Andreas system: Inferences from San Gabriel fault rock geochemistry and microstructures, J. Geophys. Res. 100, 13,007-13,020.

Favreau, P., and R. J. Archuleta (2003). Direct seismic energy modeling and application to the 1979 Imperial Valley earthquake, Geophys. Res. Lett. 30, no 5, 1198, doi 10.1029/2002GL015968. 
Freund, L. B. (1979). The mechanics of dynamic shear crack propagation, J. Geophys. Res. 84, 2199-2209.

Freund, L. B. (1990). Dynamic Fracture Mechanics, Cambridge Univ. Press, Cambridge.

Goldsby, D. L., and T. E. Tullis (2002). Low frictional strength of quartz rocks at subseismic slip rates, Geophys. Res., Lett. 29, no. 17, 1844, doi 10.1029/2002GL015240.

Guatteri, M., P. Spudich, and G. C. Beroza (2001). Inferring rate and state friction parameters from rupture models of the 1995 Hyogo-ken Nambu (Kobe) earthquake, J. Geophys. Res. 106, no. B11, 26,51126,521 .

Harris, R., and S. M. Day (1993). Dynamics of fault interactions: parallel strike-slip faults, J. Geophys. Res. 98, 4461-4472.

Harris, R., and S. M. Day (1997). Effects of a low velocity zone on a dynamic rupture, Bull. Seism. Soc. Am. 87, 1267-1280.

Heaton, T. H. (1990). Evidence for and implications of self-healing pulses of slip in earthquake rupture, Earth Planet. Int. 64, 1-20.

Hull, J. (1988). Thickness-displacement relationships for deformation zones, J. Struct. Geol. 10, 431-435.

Husseini, M. I., D. B. Jovanovich, M. J. Randall, and L. B. Freund (1975). The fracture energy of earthquakes, Geophys. J. R. Astr. Soc. 43, 367-385.

Ida, Y. (1972). Cohesive force across the tip of a longitudinal shear crack and Griffith's crack specific surface energy, J. Geophys. Res. 77, 3796-3805.

Ide, S. (2002). Estimation of radiated energy of finite-source earthquake models, Bull. Seism. Soc. Am. 92, no. 8, 2994-3005.

Johnson, E. (1990). On the initiation of unidirectional slip, Geophys. J. Int. 101, 125-132.

Kame, N., and T. Yamashita (1999). Simulation of the spontaneous growth of a dynamic crack without constraints on the crack tip path, Geophys. J. Int. 139, 345-358.

Kame, N., J. R. Rice, and R. Dmowska (2003). Effects of pre-stress and rupture velocity on dynamic fault branching, J. Geophys. Res. 108, no. B5, 2265, doi 10.1029/2002JB002189, ESE 13-1-13-21.

Lachenbruch, A. H. (1980). Frictional heating, fluid pressure, and the resistance to fault motion, J. Geophys. Res. 85, 6097-6112.

Lachenbruch, A. H., and J. H. Sass (1980). Heat flow and energetics of the San Andreas fault zone, J. Geophys. Res. 85, 6185-6222.

Li, V. C. (1987). Mechanics of shear rupture applied to earthquake zones, Fracture Mechanics of Rock, B. K. Atkinson (Editor), Academic Press, London, 351-428.

Lockner, D., H. Naka, H. Tanaka, H. Ito, and R. Ikeda (2000). Permeability and strength of core samples from the Nojima fault of the 1995 Kobe earthquake, in Proceedings of the Internaional Workshop on the Nojima Fault Core and Borehole Data Analysis, Tsukuba, Japan, 2223, November 1999, H. Ito, K. Fujimoto, H. Tanaka, and D. Lockner (Editors), U.S. Geol. Surv. Open-File Rept. 00-129, 147-152.

Mase, C. W., and L. Smith (1987). Effects of frictional heating on the thermal, hydrologic, and mechanical response of a fault, J. Geophys. Res. 92, 6249-6272.

Morrissey, J. W., and J. R. Rice (1998), Crack front waves, J. Mech. Phys. Solids 46, 467-487.

Muskhelishvili, N. I. (trans. by J. R. M. Radok) (1953). Some Basic Problems of the Mathematical Theory of Elasticity, Noordhoff, Groningen.

Nielsen, S. B., and J. M. Carlson (2000). Rupture pulse characterization; self-healing, self-similar, expanding solutions in a continuum model of fault dynamics, Bull. Seism. Soc. Am. 90, 1480-1497.

Nielsen, S., and R. Madariaga (2003). On the self-healing fracture mode, Bull. Seism. Soc. Am. 93, no. 6, 2375-2388.

Olsen, K., R. Madariaga, and R. Archuleta (1997). Three dimensional dynamic simulation of the 1992 Landers earthquake, Science 278, 834-838.

Otsuki, K., N. Monzawa, and T. Nagase (2003). Fluidization and melting of fault gouge during seismic slip: identification in the Nojima fault zone and implications for local earthquake mechanisms, J. Geophys. Res. 108, no. B4, 2192, doi 10.1029/2001JB001711, ESE 4-1-4-18.
Palmer, A. C., and J. R. Rice (1973). The growth of slip surfaces in the progressive failure of over-consolidated clay, Proc. R. Soc. London A 322, 527-548.

Perrin, G., J. R. Rice, and G. Zheng (1995). Self-healing slip pulse on a frictional surface, J. Mech. Phys. Solids 43, 1461-1495.

Peyrat, S., K. Olsen, and R. Madariaga (2001). Dynamic modeling of the 1992 Landers earthquake, J. Geophys. Res. 106, no. B11, 26,46726,482 .

Poliakov, A. N. B., R. Dmowska, and J. R. Rice (2002). Dynamic shear rupture interactions with fault bends and off-axis secondary faulting, J. Geophys. Res. 107, no. B11, 2295, doi 10.1029/2001JB000572, ESE 6-1-6-18.

Pollard, D. D., and P. Segall (1987). Theoretical displacements and stresses near fractures in rock: with applications to faults, joints, veins, dikes, and solution surfaces, in Fracture Mechanics of Rock, B. K. Atkinson (Editor), Academic Press, London, 277-349.

Power, W. L., T. E. Tullis, and J. D. Weeks (1988). Roughness and wear during brittle faulting, J. Geophys. Res. 93, 15,268-15,278.

Prasher, C. (1987). Crushing and Grinding Process Handbook, John Wiley and Sons, New York.

Rice, J. R. (1980). The mechanics of earthquake rupture, in Physics of the Earth's Interior, Proc. International School of Physics 'Enrico Fermi', A. M. Dziewonski and E. Boschi (Editors), Italian Physical Society, Bologna, and North Holland Publishing Co., Amsterdam, 555-649.

Rice, J. R. (1999). Flash heating at asperity contacts and rate-dependent friction (abstract), EOS Trans. Am. Geophys. Union 80, no. 46 (Fall Meet. Suppl.), F681.

Rice, J. R. (2000). Fracture energy of earthquakes and slip-weakening rupture parameters (abstract), EOS Trans. Am. Geophys. Union 81 (Fall Meet. Suppl.), F1227.

Rice, J. R. (2003). Earthquake fracture energies and weakening of faults by thermal pressurization of pore fluid (abstract), EOS Trans. Am. Geophys. Union 84 (Fall Meet. Suppl.), S41G-01, F1088.

Rice, J. R., and M. P. Cleary (1976). Some basic stress-diffusion solutions for fluid-saturated elastic porous media with compressible constituents, Rev. Geophys. Space Phys. 14, 227-241.

Rice, J. R., Y. Ben-Zion, and K. S. Kim (1994), Three-dimensional perturbation solution for a dynamic planar crack moving unsteadily in a model elastic solid, J. Mech. Phys. Solids 42, 813-843.

Rubin, A. M., and C. B. Parker (1994). Near-tip stress fields for dynamically propagating mode-II fractures, in The Mechanical Involvement of Fluids in Faulting, S. Hickman, R. Sibson, and R. Bruhn (Editors), U.S. Geol. Surv. Open-File Rept. 94-228, 399-405.

Rudnicki, J. W., and M. Wu (1995). Mechanics of dip-slip faulting in an elastic half-space, J. Geophys Res. 100, no. B11, 22,173-22,186.

Sammis, C.G., and S. J. Steacy (1994). The micromechanics of friction in a granular layer, Pure Appl. Geophys. 142, 777-794.

Sammis, C. G., G. C. P. King, and R. Biegel (1987). The kinematics of gouge deformation, Pure Appl. Geophys. 125, 777-812.

Scholz, C. H. (1987). Wear and gouge formation in brittle faulting, Geology 15, 493-495.

Schulz, S. E., and J. P. Evans (2000). Mesoscopic structure of the Punchbowl fault, southern California and the geologic and geophysical structure of active strike slip faults, J. Struct. Geol. 22, 913-930.

Segall, P., and D. D. Pollard (1980). Mechanics of discontinuous faults, J. Geophys. Res. 85, 4337-4350.

Segall, P., and D. D. Pollard (1983). Nucleation and growth of strike-slip faults in granite, J. Geophys. Res. 88, 555-568.

Segall, P., and J. R. Rice (1995). Dilatancy, compaction, and slip instability of a fluid infiltrated fault, J. Geophys. Res. 100, 22,155-22,171.

Sibson, R. H. (1973). Interactions between temperature and pore-fluid pressure during earthquake faulting and a mechanism for partial or total stress relief, Nature 243, no. 126, 66-68.

Sibson, R. H. (1975). Generation of pseudotachylyte by ancient seismic faulting, Geophys. J. R. Astr. Soc. 43, 775-794.

Sibson, R. H. (1986). Brecciation processes in fault zones: inferences from earthquake rupturing, Pure Appl. Geophys. 124, 159-176. 
Spray, J. G. (1993). Viscosity determinations of some frictionally generated silicate melts; implications for fault zone rheology at high strain rates, J. Geophys. Res. 98, 8053-8068.

Spray, J. G. (1995). Pseudotachylyte controversy; fact or friction?, Geology 23, 1119-1122.

Steacy, S. J., and C. G. Sammis (1991). An automaton for fractal patterns of fragmentation, Nature 353, 250-252.

Tullis, T. E., and D. Goldsby (2003), Flash melting of crustal rocks at almost seismic slip rates (abstract), EOS Trans. Am. Geophys. Union 84, no. 46 (Fall Meet. Suppl.), S51B-05, F1114.

Weertman, J. (1980). Unstable slippage across a fault that separates elastic media of different elastic-constants, J. Geophys. Res. 85, no. B3, $1455-1461$.

Wibberley, C. A. J. (2002). Hydraulic diffusivity of fault gouge zones and implications for thermal pressurization during seismic slip, Earth Planets Space 54, no. 11, 1153-1171.

Wibberley, C. A. J., and T. Shimamoto T. (2003). Internal structure and permeability of major strike-slip fault zones: the Median Tectonic Line in Mie Prefecture, Southwest Japan, J. Struct. Geol. 25, 59-78.

Wilson, J. E., J. S. Chester, and F. M. Chester (1999). Microfracture fabric of the Punchbowl Fault zone, San Andreas system, California, EOS Trans. Am. Geophys. Union 81, (Fall Meeting Suppl.), F746-F747.

Yoffe, E. H. (1951). The moving Griffith crack, Phil. Mag. 42, 739-750.

Zheng, G., and J. R. Rice (1998). Conditions under which velocity-weakening friction allows a self-healing versus a cracklike mode of rupture, Bull. Seism. Soc. Am. 88, 1466-1483.

Zoback, M. D., M. L. Zoback, V. S. Mount, J. Suppe, J. P. Eaton, J. H. Healy, D. Oppenheimer, P. Reasenberg, L. Jones, C. B. Raleigh, I. G. Wong, O. Scotti, and C. Wentworth (1988). New evidence on the state of stress of the San Andreas fault system, Science 238, 11051111.

\section{Appendix: Stresses Near a Propagating Slip Pulse}

This appendix is an extension of the appendix in Poliakov et al. (2002) (PDR) to the case of a finite sliding zone, as considered earlier by Broberg (1978) and Freund (1979). The analysis is done here for the particular slip-weakening model illustrated in Figure 2. The problem is again to find an analytic function $M(z)$ of the complex variable $z$, this time having a specified stress on the slipping portion of the $x$ axis $-L<x<0$.

For mode II

$$
\sigma_{y x}=\sigma_{y x}^{o}+\operatorname{Re}[M(x)] \text { on }-L<x<0,
$$

and, as discussed in the text, we require

$$
\begin{aligned}
& \operatorname{Re}[M(x)] \\
& \quad=\left\{\begin{array}{l}
\tau_{r}-\sigma_{y x}^{o}, \quad-L<x<-R \\
\left(1+\frac{x}{R}\right)\left(\tau_{p}-\tau_{r}\right)+\tau_{r}-\sigma_{y x}^{o}, \quad-R<x<0
\end{array}\right.
\end{aligned}
$$

For mode III, substitute $\sigma_{y z}^{o}$ for $\sigma_{y x}^{o}$.

Additional conditions imposed are that $M(z)$ vanishes as $z \rightarrow \infty$ and is bounded as $z \rightarrow 0$ and as $z \rightarrow-L$. Note that in our problem, because $\sigma_{y x}$ and $\sigma_{y z}$ are continuous across the fault,

$$
\operatorname{Re}\left[M^{+}(x)\right]=\operatorname{Re}\left[M^{-}(x)\right]=g(x)
$$

for $-\infty<x<\infty$, where $g(x)$ is the right side in (A2) for $-L<x<0$ and is $\sigma_{y x}(x, 0)-\sigma_{y x}^{o}$ for all $x$.

If we define $\bar{M}(\zeta)$ as $\overline{M(\bar{\zeta})}$, then $\bar{M}(\bar{z})=\overline{M(z)}$, and $2 \operatorname{Re}[M(z)]=M(z)+\bar{M}(\bar{z})$. Hence, $2 \operatorname{Re}\left[M^{+}(x)\right]=M^{+}(x)$ $+\bar{M}^{-}(x)=2 \operatorname{Re}\left[M^{-}(x)\right]=M^{-}(x)+\bar{M}^{+}(x)=2 g(x)$, and we find by subtraction that $[M(x)-\bar{M}(x)]^{+}=[M(x)-$ $\bar{M}(x)]^{-}$for all $x$. This means that $M(z)-\bar{M}(z)$ is actually analytic across the cut, and hence is analytic everywhere. But $M(z) \rightarrow 0$ and $\bar{M}(z) \rightarrow 0$ as $|z| \rightarrow \infty$. Thus, $M(z)-\bar{M}(z)$ $=0$ for all $z$, which means that $\bar{M}(z)=M(z)$ and $2 \operatorname{Re}[M(z)]$ $=M(z)+M(\bar{z})$. Thus, $\operatorname{Re}[M(x)]=g(x),-L<x<0$, is equivalent to the condition

$$
\mathrm{M}^{+}(x)+M^{-}(x)=2 g(x), \quad-L<x<0 .
$$

By standard methods (e.g., Muskhelishvili, 1953) we now seek a function $X(z)$, which is analytic outside the cut and satisfies $X^{+}(x)+X^{-}(x)=0,-L<x<0$, so that when we divide (A4) by $X^{+}(x)$ and use $X^{+}(x)=-X^{-}(x)$, we get

$$
\left(\frac{M(x)}{X(x)}\right)^{+}-\left(\frac{M(x)}{X(x)}\right)^{-}=\frac{2 g(x)}{X^{+}(x)}
$$

on the cut. We note also that $M(z) / X(z)$ is also analytic outside the cut if $X(z)$ has no zeros there.

Such a function that satisfies $X^{+}(x)+X^{-}(x)=0$ is $X(z)=z^{-1 / 2}(z+L)^{-1 / 2}$, where $z^{1 / 2}=\sqrt{r_{1}} e^{i \theta_{1} / 2}$, with $z=$ $r_{1} e^{i \theta_{1}}$ and $-\pi<\theta_{1}<+\pi$, and $(z+L)^{1 / 2}=\sqrt{r_{2}} e^{i \theta_{2} / 2}$, with $z+L=r_{2} e^{i \theta_{2}}$ and $-\pi<\theta_{2}<+\pi$.

The motivation for this transformation is that (A5) is in a suitable form to solve with the Cauchy integral representation. Letting $F(z)=M(z) / X(z)$ and $h(x)=2 g(x) / X^{+}(x)$, we have the problem of finding the function $F(z)$, analytic outside the cut from $-L$ to 0 , and constant at $\infty$ (because it is easy to show that our $M(z)$ is such that $z M(z)$ remains bounded as $z \rightarrow \infty)$, satisfying $F^{+}(x)-F^{-}(x)=h(x)$, a given function on $-L<x<0$. Referring to Figure A1, the Cauchy integral representation is

$$
2 \pi i F(z)=\oint_{C_{\rho}} \frac{F(t)}{t-z} d t+\oint_{C_{\mathrm{cut}}} \frac{F(t)}{t-z} d t
$$

where $C_{\text {cut }}$ is traversed in clockwise sense and $C_{\rho}$ (a large circle of radius $\rho$ ) in counter-clockwise sense, shrinking $C_{\text {cut }}$ to the slipping zone along the $x$ axis:

$$
\oint_{C_{\text {cut }}} \frac{F(t)}{t-z} d t=\int_{-L}^{0} \frac{F^{+}(t)-F^{-}(t)}{t-z} d t=\int_{-L}^{0} \frac{h(t)}{t-z} d t .
$$

Letting $\rho \rightarrow \infty$

$$
\lim _{\rho \rightarrow \infty} \oint_{C_{R}} \frac{F(t)}{t-z} d t=F(\infty) \oint_{C_{R}} \frac{d t}{t}=2 \pi i F(\infty)
$$




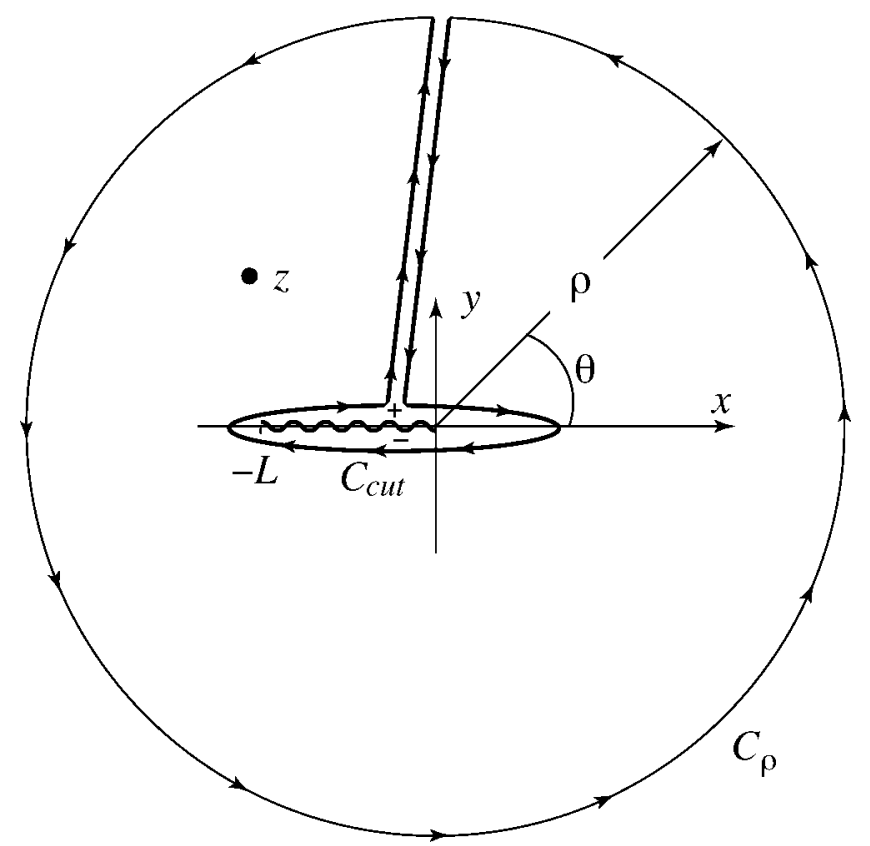

Figure A1. Integration path in complex plane, for calculation of stress field solution.

and we have

$$
F(z)=F(\infty)+\frac{1}{2 \pi i} \int_{-L}^{0} \frac{h(t)}{t-z} d t
$$

So the solution to (A5) is

$$
\frac{M(z)}{X(z)}=\frac{1}{2 \pi i} \int_{-L}^{0} \frac{2 g(t) / X^{+}(t)}{t-z} d t+\frac{A}{\pi}
$$

where $A$ is a constant. (In the next section, we show that the locked-in displacement $\delta$ across the healed part of the fault is proportional to A.) Because $X^{+}(t)=(1 / i \sqrt{-t})$ $(1 / \sqrt{t+L})$, we can write:

$$
\begin{aligned}
M(z)= & \frac{z^{-1 / 2}(z+L)^{-1 / 2}}{\pi} \\
& {\left[\int_{-L}^{0} \frac{g(t) \sqrt{-t} \sqrt{t+L}}{t-z} d t+A\right] . }
\end{aligned}
$$

To ensure no singularity at $z=0$, we must relate parameters of the model by

$$
A=\int_{-L}^{0} g(t) \sqrt{\frac{t+L}{-t}} d t
$$

and for no singularity at $z=-L$, by

$$
A=\int_{-L}^{0} g(t) \sqrt{\frac{-t}{t+L}} d t
$$

Using (A2) for $g(x)$ in (A7),

$$
\begin{array}{r}
A=-\left(\sigma_{x y}^{o}-\tau_{r}\right) \int_{-L}^{0} \sqrt{\frac{t+L}{-t}} d t+\left(\tau_{p}-\tau_{r}\right) \\
\int_{-R}^{0}\left(1+\frac{t}{R}\right) \sqrt{\frac{t+L}{-t}} d t .
\end{array}
$$

Substituting $t=-(L / 2)+(L / 2) \cos \theta, d t=-(L / 2) \sin$ $\theta d \theta$ and integrating yields (the following has been simplified by using equation A14 subsequently, a consequence of equations $\mathrm{A} 7$ and $\mathrm{A} 8$ ):

$$
A=R\left(\tau_{p}-\tau_{r}\right) \frac{\theta^{\prime}-\sin \theta^{\prime} \cos \theta^{\prime}}{8 \sin ^{4}\left(\theta^{\prime} / 2\right)},
$$

where $\theta^{\prime}=2 \sin ^{-1}(\sqrt{R / L})$. For consistency, the two values of $A$ in (A7) and (A8) must be the same, giving the condition

$$
\begin{aligned}
\int_{-L}^{0} g(t)\left(\sqrt{\frac{t+L}{-t}}\right. & \left.+\sqrt{\frac{-t}{t+L}}\right) d t \\
& \equiv L \int_{-L}^{0} \frac{g(t)}{\sqrt{-t(t+L)}} d t=0
\end{aligned}
$$

Now, combining (A6) and (A7), and using

$$
\frac{\sqrt{-t}}{t-z}+\frac{1}{\sqrt{-t}}=-\frac{z}{\sqrt{-t}(t-z)}
$$

(A6) becomes

$$
M(z)=-\frac{1}{\pi}\left(\frac{z}{z+L}\right)^{1 / 2} \int_{-L}^{0} \frac{g(t)}{t-z} \sqrt{\frac{t+L}{-t}} d t .
$$

Similarly, after noting that we can add the integrand of equation (A10) to this (thus removing the apparent singularity at the trailing edge of the slip pulse) and using the algebraic manipulation

$$
\frac{\sqrt{t+L}}{t-z}-\frac{1}{\sqrt{t+L}}=\frac{z+L}{(t-z) \sqrt{t+L}},
$$


we obtain

$$
\begin{array}{r}
M(z)=-\frac{1}{\pi} z^{1 / 2}(z+L)^{1 / 2} \\
\int_{-L}^{0} \frac{g(t)}{\sqrt{-t(t+L)}} \frac{d t}{(t-z)},
\end{array}
$$

which is of the same form as an analogous representation given by Broberg (1978). Thus, substituting $t=-(L / 2)$ $(1-\cos \theta)$,

$$
\begin{aligned}
M(z)=-\frac{1}{\pi} z^{1 / 2}(z+L)^{1 / 2} & \\
& \int_{0}^{\pi} \frac{g[t(\theta)] d \theta}{(L / 2) \cos \theta-(z+L / 2)} .
\end{aligned}
$$

where

$$
g[t(\theta)]=\left\{\begin{array}{r}
-\left(\sigma_{y x}^{o}-\tau_{r}\right), \quad \theta^{\prime}<\theta<\pi \\
-\left(\sigma_{y x}^{o}-\tau_{r}\right)+\left(\tau_{p}-\tau_{r}\right)\left[1-\frac{L}{2 R}(1-\cos \theta)\right], \\
0<\theta<\theta^{\prime}
\end{array}\right.
$$

where, from (A10), the parameters must be related by $\int_{0}^{\pi} g[t(\theta)] d \theta=0$. Since $t=-(L / 2)(1-\cos \theta)$ gives $-R=-(L / 2)\left(1-\cos \theta^{\prime}\right)$, or $R / L=\sin ^{2}\left(\theta^{\prime} / 2\right)$, the condition (A10) gives

$$
\frac{\sigma_{y x}^{o}-\tau_{r}}{\tau_{p}-\tau_{r}}=\frac{\theta^{\prime}}{\pi}-\frac{\theta^{\prime}-\sin \theta^{\prime}}{2 \pi \sin ^{2}\left(\theta^{\prime} / 2\right)} .
$$

for mode II. The same equation holds for $\left(\sigma_{y z}^{o}-\tau_{r}\right) /\left(\tau_{p}-\right.$ $\tau_{r}$ ) in mode III. Note that this solution is only valid for $0<$ $\theta^{\prime}<\pi$, which imposes an upper limit on the scaled stress $\operatorname{drop}\left(\sigma_{y x}^{o}-\tau_{r}\right) /\left(\tau_{p}-\tau_{r}\right)=1 / 2$. It is possible that healing occurs before the strength drop is complete and $R>L$, but we will not consider that case here.

It is convenient to divide the integral (A12) into two parts writing $M(z)=M^{0}(z)+M^{1}(z)$, where $M^{0}(z)$ corresponds to the constant part of $g(t)$ in (A13) and $M^{1}(z)$ to the part of $g(t)$ that depends on $\cos \theta$.

$$
\begin{aligned}
M^{0}(z)= & \frac{\left(\sigma_{y x}^{o}-\tau_{r}\right)}{\pi} z^{1 / 2}(z+L)^{1 / 2} \int_{-L}^{0} \frac{d t}{\sqrt{-t(t+L)}(t-z)} \\
= & -\frac{\left(\sigma_{y x}^{o}-\tau_{r}\right)}{\pi i} \frac{1}{X(x)} \int_{-L}^{0} \frac{X^{+}(t)}{(t-z)} d t \\
= & -\frac{\left(\sigma_{y x}^{o}-\tau_{r}\right)}{2 \pi i} \frac{1}{X(z)} \oint_{C_{\mathrm{cut}}} \frac{X(t)}{(t-z)} d t .
\end{aligned}
$$

As earlier for $F(z)$ (see Fig. A1), $\oint_{C_{\rho}} \frac{X(t)}{(t-z)} d t+$ $\oint_{C_{\mathrm{cut}}} \frac{X(t)}{(t-z)} d t=2 \pi i X(z)$. However, $\lim _{\rho \rightarrow \infty} \oint_{C_{\rho}} \frac{X(t)}{(t-z)} d t=0$, and hence $\oint_{C_{\mathrm{cut}}} \frac{X(t)}{(t-z)} d t=2 \pi i X(z)$. Thus, $M^{0}(z)$ is independent of $z$, and $M^{0}(z)=-\left(\sigma_{y x}^{o}-\tau_{r}\right)$. Using (A14), we can rewrite this as

$$
M^{0}(z)=-\left(\tau_{p}-\tau_{r}\right)\left[\frac{\theta^{\prime}}{\pi}-\frac{\theta^{\prime}-\sin \theta^{\prime}}{2 \pi \sin ^{2}\left(\theta^{\prime} / 2\right)}\right] .
$$

We now turn our attention to $M^{1}(z)$.

$$
\begin{aligned}
M^{1}(z)= & \frac{\left(\tau_{p}-\tau_{r}\right) z^{1 / 2}(z+L)^{1 / 2}}{\pi} \int_{0}^{\theta^{\prime}} \frac{[1-(L / 2 R)(1-\cos \theta)]}{(z+L / 2)-(L / 2) \cos \theta} d \theta \\
= & -\frac{\left(\tau_{p}-\tau_{r}\right) z^{1 / 2}(z+L)^{1 / 2}}{\pi} \\
& {\left[\frac{2}{L}\left(1+\frac{z}{R}\right) \int_{0}^{\theta^{\prime}} \frac{d \theta}{\cos \theta-1-2 z / L}+\frac{\theta^{\prime}}{R}\right] }
\end{aligned}
$$

We thus have an integral of the form $I(c)=\int_{0}^{\theta^{\prime}} \frac{d \theta}{\cos \theta-c}$, where $c=1+2 z / L$. Making the change of variables $\sigma=$ $\exp (i \theta)$,

$$
\begin{aligned}
I(c)= & \frac{1}{i} \int_{1}^{\exp \left(i \theta^{\prime}\right)} \frac{d \sigma}{\sigma[(\sigma+1 / \sigma) / 2-c]} \\
= & \frac{2}{i} \int_{1}^{\exp \left(i^{\prime}\right)} \frac{d \sigma}{\left(\sigma-\sigma_{1}\right)\left(\sigma-\sigma_{2}\right)} \\
& =\frac{1}{i \sqrt{c^{2}-1}} \ln \left(\frac{e^{i \theta^{\prime}}-\sigma_{1}}{1-\sigma_{1}} \times \frac{1-\sigma_{2}}{e^{i \theta^{\prime}}-\sigma_{2}}\right),
\end{aligned}
$$

where $\sigma_{1}=c+\sqrt{c^{2}-1}$ and $\sigma_{2}=c-\sqrt{c^{2}-1}$ are the roots of $(1 / 2)\left(\sigma^{2}+1\right)-c \sigma$. Thus,

$$
\begin{gathered}
M^{1}(z)=-\frac{1}{\pi}\left(\tau_{p}-\tau_{r}\right)\left\{\left(1+\frac{z}{R}\right)\left[\frac{1}{i} \ln (F(z))\right]\right. \\
\left.+\frac{z^{1 / 2}(z+L)^{1 / 2} \theta^{\prime}}{R}\right\},
\end{gathered}
$$

where

$$
\begin{array}{r}
F(z)=\left(\frac{\left(e^{i \theta^{\prime}}-1\right) L / 2-z-z^{1 / 2}(z+L)^{1 / 2}}{\left(e^{i \theta^{\prime}}-1\right) L / 2-z+z^{1 / 2}(z+L)^{1 / 2}}\right) \\
\left(\frac{z-z^{1 / 2}(z+L)^{1 / 2}}{z+z^{1 / 2}(z+L)^{1 / 2}}\right),
\end{array}
$$


and finally, (A15) and (A17) are combined to give $M(z)=$ $M^{0}(z)+M^{1}(z)$.

Two consistency checks are satisfied, as follows:

1. As $z / L \rightarrow 0$ and $R / L \rightarrow 0$ at fixed $z / R$, this approaches the solution in PDR.

2. As $z / L \rightarrow \infty$ for any fixed $R / L$, this $M(z) \rightarrow 0$.

\section{The Total Locked-in Displacement Produced by a Slip Pulse}

The total locked-in displacement, $\delta=u_{x}^{+}-u_{x}^{-}$, produced by a slip pulse at $x<-L$ can be found using the expressions for displacements given by PDR which we use to write:

$$
u_{x}=\operatorname{Re}\left[\frac{2 \alpha_{s}}{i \mu D} \int^{z_{d}} M(z) d z-\frac{\left(1+\alpha_{s}^{2}\right) \alpha_{s}}{i \mu D} \int^{z_{s}} M(z) d z\right]
$$

In the limit $|z| \gg L$, equation (A6) becomes $M(z)=A / \pi z$, so integration in (A18) gives, where $|z| \gg L$ :

$$
\begin{gathered}
u_{x}=\frac{A}{\pi} \operatorname{Re}\left[\frac{2 \alpha_{s}}{i \mu D} \ln \left(z_{d}\right)-\frac{\left(1+\alpha_{s}^{2}\right) \alpha_{s}}{i \mu D} \ln \left(z_{s}\right)\right] \\
=\frac{2 \alpha_{s} A}{\mu D \pi}\left(\theta_{d}-\frac{1+\alpha_{s}^{2}}{2} \theta_{s}\right) .
\end{gathered}
$$

That uses $\ln (z)=\ln (r)+i \theta$; the real parts of (A19) involve just the theta terms. Because both theta change by $2 \pi$ in going from the lower to the upper surfaces of the region that has been slipped and is now locked again, we thus end up with

$$
\delta=u_{x}^{+}-u_{x}^{-}=2 A \alpha_{s}\left(1-\alpha_{s}^{2}\right) / \mu D
$$

showing the proportionality of $A$ to $\delta$. Substituting (A9) for $A$ then gives

$$
\delta=\frac{\left(\tau_{p}-\tau_{r}\right) R}{\mu}\left(\frac{\theta^{\prime}-\sin \theta^{\prime} \cos \theta^{\prime}}{4 \sin ^{4}\left(\theta^{\prime} / 2\right)}\right) \frac{\alpha_{s}\left(1-\alpha_{s}^{2}\right)}{D} .
$$

The same applies for mode III, but with the last fraction replaced by $1 / \alpha_{s}$.

\section{The Slip-Weakening Zone Length $R$}

The basic energy balance for the slip pulse is

$$
\begin{aligned}
\sigma_{y x}^{o} \delta=\int_{0}^{\delta} \tau(\Delta u) d(\Delta u)=\int_{0}^{\delta}[\tau(\Delta u) \\
\left.-\tau_{r}\right] d(\Delta u)+\tau_{r} \delta=G+\tau_{r} \delta
\end{aligned}
$$

The result follows because $\sigma_{y x}^{o} \delta$ is the work done by applied stresses per unit area of advance of the rupture front. That work goes into changes of strain and kinetic energy, of which there are none in this steady-state configuration, and into frictional dissipation, which is $\int_{0}^{\delta} \tau(\Delta u) d(\Delta u)$ per unit of new ruptured area. This shows that

$$
\delta=G /\left(\sigma_{y x}^{o}-\tau_{r}\right),
$$

and hence that $\delta$ is independent of rupture speed in this model, for given fracture energy and stress drop. The expression may be rearranged, with use of earlier expressions, to obtain an expression for $R$. Let us write it as

$$
G=\left(\sigma_{y x}^{o}-\tau_{r}\right) \delta=\left(\frac{\sigma_{y x}^{o}-\tau_{r}}{\tau_{p}-\tau_{r}}\right)\left(\tau_{p}-\tau_{r}\right) \delta
$$

Substituting (A14) for the ratio stress drop/strength drop (the first term on the right) and (A21) for $\delta$ gives

$$
G=\frac{h\left(\theta^{\prime}\right) \alpha_{s}\left(1-\alpha_{s}^{2}\right)}{D} \frac{\left(\tau_{p}-\tau_{r}\right)^{2} R}{\mu},
$$

where

$$
h\left(\theta^{\prime}\right)=\left(\frac{\theta^{\prime}}{\pi}-\frac{\theta^{\prime}-\sin \theta^{\prime}}{2 \pi \sin ^{2}\left(\theta^{\prime} / 2\right)}\right)\left(\frac{\theta^{\prime}-\sin \theta^{\prime} \cos \theta^{\prime}}{4 \sin ^{4}\left(\theta^{\prime} / 2\right)}\right) .
$$

Hence, we have for the slip-weakening zone length

$$
R=\frac{D}{h\left(\theta^{\prime}\right) \alpha_{s}\left(1-\alpha_{s}^{2}\right)} \frac{\mu G}{\left(\tau_{p}-\tau_{r}\right)^{2}}
$$

All expressions in this section apply also for mode III when we replace $\sigma_{y x}^{o}$ with $\sigma_{y z}^{o}$ and replace the ratio $D /\left[\alpha_{s}\left(1-\alpha_{s}^{2}\right)\right]$ in (A24) and (A26) with $\alpha_{s}$. Both of those velocitydependent terms are called $F\left(v_{r}\right)$ in the text of the article.

\section{Slip-Weakening Law Implied by the Assumed Stress Distribution}

Using Equation (A18), we can solve for the slip $\Delta u(x)$ $=u_{x}^{+}(x, 0)-u_{x}^{-}(x, 0)$ along the sliding part of the rupture, $-L<x<0$. Because we have assumed a form for the stress variation there (Fig. 2), a slip-weakening law is implied. From (A18) we obtain

$$
\begin{aligned}
\Delta u(x)=\frac{\left(1-\alpha_{s}^{2}\right) \alpha_{s}}{\mu D} \int_{x}^{0} \operatorname{Im}[ & M\left(x+i 0^{-}\right) \\
& \left.-M\left(x+i 0^{+}\right)\right] d x .
\end{aligned}
$$


The same applies for mode III when the factor in front is replaced by $1 /\left(\mu \alpha_{s}\right)$; in that case, $\Delta u(x)=u_{z}^{+}(x, 0)-$ $u_{z}^{-}(x, 0)$. From equations (6) and (7), the structure of the integrand is of the form:

$$
\begin{aligned}
\operatorname{Im}\left[M\left(x+i 0^{-}\right)-M\left(x+i 0^{+}\right)\right] & \\
& =\left(\tau_{p}-\tau_{r}\right) m(1+x / r, R / L),
\end{aligned}
$$

where $m(1+x / R, R / L)$ (the 1 is added for convenience) is a dimensionless function of its two arguments. For our assumed stress distribution, when $x$ is in the slip-weakening zone $-R<x<0,1+x / R=T$, where $T \equiv\left(\tau-\tau_{r}\right) /\left(\tau_{p}\right.$ $-\tau_{r}$ ) is just the scaled strength drop. Thus, changing variables from $x$ to $T$ in (A27), the slip-weakening relation is described by

$$
\Delta u=\frac{\left(\tau_{p}-\tau_{r}\right) R\left(1-\alpha_{s}^{2}\right) \alpha_{s}}{\mu D} \int_{T}^{1} m\left(T^{\prime}, R / L\right) d T^{\prime} .
$$

Equation (A26) now reveals that the factor in front of the integral is just $G /\left(\tau_{p}-\tau_{r}\right)$ times a function of $\theta^{\prime}$, hence of $R / L$. Absorbing that factor into $m$, to convert it to a new dimensionless function $\hat{m}(T, R / L)$, we therefore determine the slip-weakening law $\tau=\tau(\Delta u)$ by

$$
\begin{aligned}
\Delta \mathrm{u}=\frac{G}{\left(\tau_{p}-\tau_{r}\right)} \int_{T}^{1} \hat{m}\left(T^{\prime}, R / L\right) d T^{\prime}, \\
\text { where } T=\frac{\tau(\Delta u)-\tau_{r}}{\tau_{p}-\tau_{r}} .
\end{aligned}
$$

The result is the same for modes II and III, and all dependence on $v_{r}$ disappears from it, but there is a dependence on one additional variable, namely $R / L$. The expression is difficult to evaluate, in general, but to see how significant that dependence on $R / L$ is, we compare the implied slipweakening laws in the limit cases $R / L \rightarrow 0$ and $R / L=1$. The result for $R / L \rightarrow 0$ confirms the result of Palmer and Rice (1973) and Rice (1980), and is

$$
\begin{aligned}
\Delta u=\frac{9 G}{8\left(\tau_{p}-\tau_{r}\right)}[(2-T) \sqrt{1-T} & \\
& \left.-\frac{1}{2} T^{2} \ln \left(\frac{1+\sqrt{1-T}}{1-\sqrt{1-T}}\right)\right],
\end{aligned}
$$

whereas for $R / L=1$ we find that

$$
\begin{aligned}
\Delta u=\frac{G}{\left(\tau_{p}-\tau_{r}\right)}[1- & \frac{2}{\pi} \arcsin (2 T-1) \\
& \left.-\frac{4}{\pi}(2 T-1) \sqrt{T(1-T)}\right] .
\end{aligned}
$$

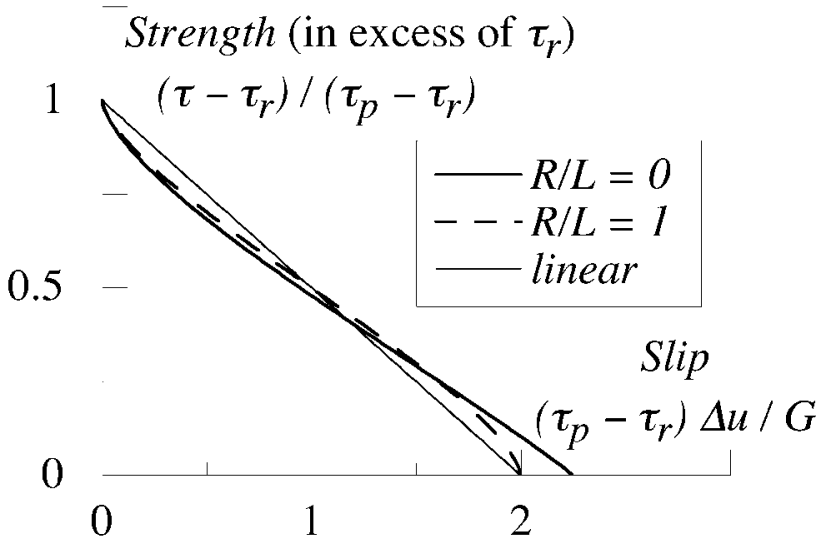

Figure A2. The slip-weakening law implied by our procedures is independent of rupture propagation speed but has a weak dependence on $R / L$ (or, equivalently, on the scaled dynamic stress drop of Fig. 3). Comparison here is of the law for $R / L=0$ and 1 . The commonly adopted linear slip-weakening law is also shown.

These define the slip-weakening laws $\tau=\tau(\Delta u)$ for $\tau_{p} \geq \tau$ $>\tau_{r}$; in the model it is assumed that $\tau(\Delta u)=\tau_{r}$ for $\Delta u$ greater than the value produced by the preceding expressions as $T \rightarrow 0^{+}$. Results of (A29) and (A30) are plotted in Figure A2. The resulting functions $\tau=\tau(\Delta u)$ differ by only a small amount from one another, despite the significantly different $R / L$; in fact, they differ by only a minor amount from the commonly adopted law that weakens linearly in $\Delta u$, shown there too. Thus, we conclude that the slip-pulse solutions devised by the methods of this article describe response to the same slip-weakening law, to a very good approximation, irrespective of crack speed and the level of dynamic stress drop.

Department of Earth and Planetary Sciences and Division of Engineering and Applied Sciences

224 Pierce Hall, 29 Oxford Street

Harvard University

Cambridge, Massachusetts 02138

rice@esag.harvard.edu

(J.R.R.)

Department of Earth Sciences

University of Southern California

Los Angeles, California 90089-0740

sammis@usc.edu

(C.G.S.)

Division of Engineering and Applied Sciences

Harvard University

Cambridge, Massachusetts 02138

rparsons@post.harvard.edu

(R.P.) 